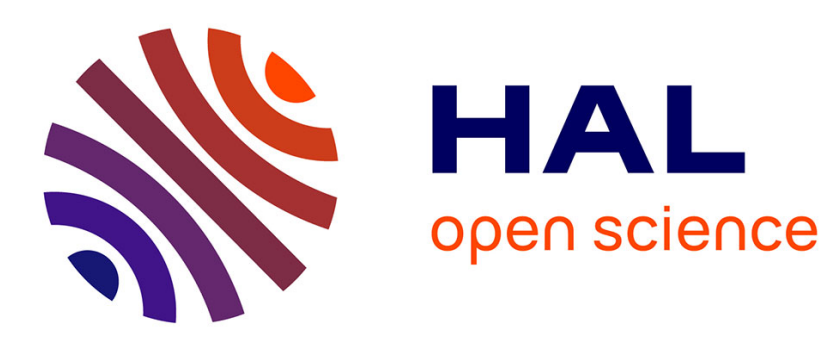

\title{
Estimating functions for SDE driven by stable Lévy processes
}

Emmanuelle Clément, Arnaud Gloter

\section{To cite this version:}

Emmanuelle Clément, Arnaud Gloter. Estimating functions for SDE driven by stable Lévy processes. 2018. hal-01570175v2

\section{HAL Id: hal-01570175 \\ https://hal.science/hal-01570175v2}

Preprint submitted on 14 Jun 2018

HAL is a multi-disciplinary open access archive for the deposit and dissemination of scientific research documents, whether they are published or not. The documents may come from teaching and research institutions in France or abroad, or from public or private research centers.
L'archive ouverte pluridisciplinaire HAL, est destinée au dépôt et à la diffusion de documents scientifiques de niveau recherche, publiés ou non, émanant des établissements d'enseignement et de recherche français ou étrangers, des laboratoires publics ou privés. 


\title{
Estimating functions for SDE driven by stable Lévy processes
}

\author{
Emmanuelle Clément ${ }^{\mathrm{a}}$ and Arnaud Gloter ${ }^{\mathrm{b}}$ \\ a Laboratoire MICS, Fédération de Mathématiques FR 3487 \\ Université Paris-Saclay, CentraleSupélec \\ 91190 Gif-sur-Yvette, France \\ E-mail: emmanuelle.clement@u-pem.fr \\ ' LaMME, Université d'Evry, CNRS \\ Université Paris-Saclay \\ 91025 Evry, France \\ E-mail: arnaud.gloter@univ-evry.fr
}

\begin{abstract}
This paper is concerned with parametric inference for a stochastic differential equation driven by a purejump Lévy process, based on high frequency observations on a fixed time period. Assuming that the Lévy measure of the driving process behaves like that of an $\alpha$-stable process around zero, we propose an estimating functions based method which leads to asymptotically efficient estimators for any value of $\alpha \in(0,2)$ and does not require any integrability assumptions on the process. The main limit theorems are derived thanks to a control in total variation distance between the law of the normalized process, in small time, and the $\alpha$-stable distribution. This method is an alternative to the non Gaussian quasi-likelihood estimation method proposed by Masuda [21] where the Blumenthal-Getoor index $\alpha$ is restricted to belong to the interval [1,2).
\end{abstract}

MSC 2010 subject classifications: Primary 60G51, 60G52, 60J75, 62F12; secondary 60H07, 60F05 .

Keywords: Lévy process, Stable process, Stochastic Differential Equation, Parametric inference, Estimating functions, Malliavin Calculus.

\section{Introduction}

Pure-jump processes are widely used and appropriate in several fields such as traffic modeling, energy market modeling and estimation of such processes is a currently active topic. In this paper we are interested in parametric estimation of the drift and scale coefficients for a one-dimensional stochastic differential equation

$$
d X_{t}=b\left(X_{t}, \theta\right) d t+a\left(X_{t-}, \sigma\right) d L_{t},
$$

where $L$ is a locally stable pure-jump Lévy process with Blumenthal-Getoor index $\alpha \in(0,2)$. This choice of $L$ encompasses stable processes and also many other interesting processes.

We study the estimation of $(\theta, \sigma)$ from discrete equidistant observations of $X$ on a fixed time interval with time grid mesh shrinking to zero. In this high-frequency observation context it is known that the estimation of $\theta$ is impossible if the driving process $L$ contains a Brownian part (which corresponds to the case $\alpha=2$ ). However when $\alpha<2$, both parameters $\theta$ and $\sigma$ can be estimated. This problem has been studied first when

${ }^{\mathrm{a} C}$ Corresponding author 
$X$ is a Lévy process (which corresponds to constant coefficients $a$ and $b$ ) in several papers, see for example Aït-Sahalia and Jacod [1] [2], Kawai and Masuda [15] [16], Masuda [20], Ivanenko, Kulik and Masuda [11]. In all these papers, the increments of the observed process $X$ are independent with an explicit characteristic function. However, contrarily to the jump-diffusion case, for a general pure-jump driven SDE the literature is much smaller. It has been established recently in Clément and Gloter [7], Clément, Gloter and Nguyen [8] that the Local Asymptotic Mixed Normality property holds when the scale coefficient $a$ is assumed to be constant and $L$ is a truncated $\alpha$-stable process. This result permits to identify the Fisher information matrix for the parameters $(\theta, \sigma)$ and to show that the rate of convergence is $n^{1 / \alpha-1 / 2}$ for the estimation of $\theta$ and $n^{1 / 2}$ for the estimation of $\sigma$. It is important to remark that the estimation rate for $\theta$ is slower than the usual rate $n^{1 / 2}$ when $\alpha>1$ and faster when $\alpha<1$. Concerning the estimation problem, this has been addressed by Masuda [21] assuming that $\alpha \in[1,2)$ using a quasi-likelihood estimation method. Indeed, in that case, the drift contribution is negligible compared to the jump part and the law of the normalized increment $h^{-1 / \alpha}\left(X_{t+h}-X_{t}-h b\left(X_{t}, \theta\right)\right) / a\left(X_{t}, \sigma\right)$ is close to the $\alpha$-stable distribution as $h$ goes to zero. However, this method does not seem to be extended to the case $\alpha \in(0,1)$ mainly because of the great contribution of the drift.

In this paper, we propose an estimating functions based method which applies to any value of $\alpha \in(0,2)$. Estimating equation methods are useful alternative methods in situations where the likelihood function is not known in a tractable form and have been widely used in estimating diffusion processes from discrete time observations (see for example Bibby and Sørensen [5], Kessler and Sørensen [17]). We also refer to the papers by Barndorff-Nielsen and Sørensen [4], Sørensen [23], Jacod and Sørensen [14] for general asymptotic results on estimating equation methods. In this work, we consider estimating equations derived by approximating the score function and by changing the above normalized increment by $h^{-1 / \alpha}\left(X_{t+h}-\xi_{h}^{X_{t}}(\theta)\right) / a\left(X_{t}, \sigma\right)$, where $\left(\xi_{t}^{x}(\theta)\right)_{t}$ solves the ordinary differential equation

$$
\xi_{t}^{x}(\theta)=x+\int_{0}^{t} b\left(\xi_{s}^{x}(\theta), \theta\right) d s .
$$

The introduction of this ordinary differential equation is convenient for dealing with any value of $\alpha$ but we can also replace it by a numerical approximation scheme whose order has to be high when $\alpha$ is small. Conditionally on $X_{t}$ the density of $h^{-1 / \alpha}\left(X_{t+h}-\xi_{h}^{X_{t}}(\theta)\right) / a\left(X_{t}, \sigma\right)$ converges when $h$ tends to zero to the density of the $\alpha$-stable distribution. This has been established by Kulik [18] when $L$ is an $\alpha$-stable process (and also in [9] when $L$ is a truncated $\alpha$-stable process assuming that $a$ is constant). However to prove consistency and asymptotic mixed normality of our estimators, the convergence of the densities is not sufficient and we also need a rate of convergence. This is the most technical part of the paper. For $L$ a locally $\alpha$-stable process, we prove that the total variation distance between the conditional law of $h^{-1 / \alpha}\left(X_{t+h}-\right.$ $\left.\xi_{h}^{X_{t}}(\theta)\right) / a\left(X_{t}, \sigma\right)$ and the $\alpha$-stable distribution is bounded by $\varepsilon_{h}$ such that $h^{-1 / 2} \varepsilon_{h}$ tends to zero. This result is the key ingredient to derive some limit theorems (Law of Large Numbers, Central Limit Theorem) for functionals of normalized discrete time observations of the process $X$.

At last, it should be noted that the estimation method proposed in this paper requires that the BlumenthalGetoor index $\alpha$ is known. This is also the case in Masuda [21]. A large literature is devoted to the estimation of the jump activity of jump-diffusion processes from high frequency observations, based on truncated power variation or on empirical characteristic function. We mention among others the papers by Ait-Sahalia and Jacod [3], Todorov [24], Todorov and Tauchen [25].

This paper is organized as follows. Section 2 introduces the notations and assumptions. Section 3 is devoted to the estimating function method and states consistency and asymptotic mixed normality of our estimators after establishing some limit theorems. Section 4 is dedicated to some critical total variation distance estimates. Section 5 contains the proof of limit theorems stated in Section 3. 


\section{Notation and setup}

We consider the stochastic differential equation :

$$
X_{t}=x_{0}+\int_{0}^{t} b\left(X_{s}, \theta\right) d s+\int_{0}^{t} a\left(X_{s-}, \sigma\right) d L_{s}, \quad t \in[0,1]
$$

where $a$ and $b$ are known functions from $\mathbb{R} \times \mathbb{R}$ to $\mathbb{R}$ and $(\theta, \sigma)$ are real parameters. We assume that $\left(L_{t}\right)_{t \in[0,1]}$ is a pure-jump Lévy process defined on a filtered space $\left(\Omega, \mathcal{F},\left(\mathcal{F}_{t}\right), \mathbb{P}\right)$.

We introduce also the ordinary differential equation

$$
\xi_{t}^{x_{0}}(\theta)=x_{0}+\int_{0}^{t} b\left(\xi_{s}^{x_{0}}(\theta), \theta\right) d s, \quad t \in[0,1]
$$

It will be shown in Section 4 that if $\left(L_{t}\right)_{t \in[0,1]}$ is a locally $\alpha$-stable process with $\alpha \in(0,2)$, then the distribution of $n^{1 / \alpha}\left(X_{1 / n}-\xi_{1 / n}^{x_{0}}(\theta)\right) / a\left(x_{0}, \sigma\right)$ is close to the distribution of $n^{1 / \alpha} L_{1 / n}$.

We observe the process on discrete times $t_{i}=i / n$ for $i=1, \ldots n$ for the value $\left(\theta_{0}, \sigma_{0}\right)$ of the parameter and based on these observations our aim is to estimate $\left(\theta_{0}, \sigma_{0}\right)$.

We make the following assumptions.

H1(Regularity) : (a) Let $V_{\theta_{0}} \times V_{\sigma_{0}}$ be a neighborhood of $\left(\theta_{0}, \sigma_{0}\right)$. We assume that $x \mapsto a\left(x, \sigma_{0}\right)$ is $\mathcal{C}^{2}$ on $\mathbb{R}, b$ is $\mathcal{C}^{2}$ on $\mathbb{R} \times V_{\theta_{0}}$ and

$$
\begin{gathered}
\sup _{x}\left(\sup _{\theta \in V_{\theta_{0}}}\left|\partial_{x} b(x, \theta)\right|+\left|\partial_{x} a\left(x, \sigma_{0}\right)\right|\right) \leq C, \\
\left|\partial_{x}^{2} b\left(x, \theta_{0}\right)\right|+\left|\partial_{x}^{2} a\left(x, \sigma_{0}\right)\right| \leq C\left(1+|x|^{p}\right), \quad \text { for some } p>0, \\
\forall x \in \mathbb{R}, \forall \sigma \in V_{\sigma_{0}} \quad a(x, \sigma)>0 \quad \text { and } \sup _{\sigma \in V_{\sigma_{0}}} \frac{1}{a(x, \sigma)} \leq C\left(1+|x|^{p}\right), \quad \text { for some } p>0 .
\end{gathered}
$$

(b) $\forall x \in \mathbb{R}, \theta \mapsto b(x, \theta)$ and $\sigma \mapsto a(x, \sigma)$ are $\mathcal{C}^{3}$ and

$$
\begin{gathered}
\sup _{(\theta, \sigma) \in V_{\theta_{0}} \times V_{\sigma_{0}}} \max _{1 \leq l \leq 3}\left(\left|\partial_{\theta}^{l} b(x, \theta)\right|+\left|\partial_{\sigma}^{l} a(x, \sigma)\right|\right) \leq C\left(1+|x|^{p}\right), \quad \text { for some } p>0, \\
\sup _{\theta \in V_{\theta_{0}}}\left|\partial_{x} \partial_{\theta} b(x, \theta)\right| \leq C\left(1+|x|^{p}\right), \quad \text { for some } p>0 .
\end{gathered}
$$

Under the boundedness assumption on the derivative with respect to $x$, the coefficients $a$ and $b$ are globally Lipschitz and equation (2.1) admits a unique strong solution.

H2 (Lévy measure) : (a) The Lévy measure of $\left(L_{t}\right)$ satisfies $\nu(d z)=\frac{g(z)}{|z|^{\alpha+1}} 1_{\mathbb{R} \backslash\{0\}}(z) d z$, where $\alpha \in(0,2)$ and $g: \mathbb{R} \mapsto \mathbb{R}$ is a continuous symmetric non negative bounded function with $g(0)=1$. $\infty$.

(b) $g$ is differentiable on $\{0<|z| \leq \eta\}$ for some $\eta>0$ with continuous derivative such that $\sup _{0<|z| \leq \eta}\left|\frac{g^{\prime}}{g}\right|<$

This assumption is satisfied by a large class of processes : $\alpha$-stable process $(g=1)$, truncated $\alpha$-stable process ( $g=\tau$ a truncation function), tempered stable process $\left(g(z)=e^{-\lambda|z|}, \lambda>0\right)$.

H3 (Non degeneracy) : Almost surely, $\exists t_{1}, t_{2} \in(0,1)$, such that $\partial_{\sigma} a\left(X_{t_{1}}, \sigma_{0}\right) \neq 0, \partial_{\theta} b\left(X_{t_{2}}, \theta_{0}\right) \neq 0$, where $\left(X_{t}\right)_{t \in[0,1]}$ solves $(2.1)$ for the value $\left(\theta_{0}, \sigma_{0}\right)$ of the parameter.

We will use the following notation. By $\|$.$\| , we denote a vector norm or a matrix norm, A^{T}$ is the transpose of a matrix $A$, and for a bounded function $f: \mathbb{R} \mapsto \mathbb{R}$, we set $\|f\|_{\infty}=\sup _{x}|f(x)|$. In the sequel, we denote by $a^{\prime}, b^{\prime}$ the derivative of $a, b$ with respect to $x, \dot{a}, \dot{b}$ the derivative with respect to the parameter and $\ddot{a}, \ddot{b}$ the second order derivative with respect to the parameter. We will also use the notation $\xi_{i}(\theta)=\xi_{\frac{1}{n}}^{X_{i / n}}(\theta)$, for $i=0$ to $n$.

Throughout the paper, $C$ or $C_{p}$ denote some constants whose value does not depend on $n$ and may change from line to line. 


\section{Estimating function method and main statistical results}

To estimate $(\theta, \sigma)$, we will use the estimating function method (see for example [14] and [23]). To this end, we consider the functions

$$
G_{n}(\theta, \sigma)=\left(\begin{array}{c}
G_{n}^{1}(\theta, \sigma) \\
G_{n}^{2}(\theta, \sigma)
\end{array}\right)
$$

with for $k=1,2$

$$
G_{n}^{k}(\theta, \sigma)=\sum_{i=1}^{n} g^{k}\left(X_{\frac{i-1}{n}}, X_{\frac{i}{n}}, \theta, \sigma\right)
$$

where $g^{k}$ will be specified below. An estimator of $(\theta, \sigma)$ is obtained by solving $G_{n}(\theta, \sigma)=0$ and the properties of this estimator depend on the choice of the functions $g^{1}$ and $g^{2}$.

Denoting by $p_{1 / n}(x, y)$ the transition density of the Markov chain $\left(X_{i / n}\right)_{i}$ solution of $(2.1)$, we can prove the convergence as $n$ goes to infinity

$$
\frac{a(x, \sigma)}{n^{1 / \alpha}} p_{1 / n}\left(x, \frac{a(x, \sigma)}{n^{1 / \alpha}} y+\xi_{1 / n}^{x}(\theta)\right) \longrightarrow \varphi_{\alpha}(y),
$$

where $\varphi_{\alpha}$ is the density of $L_{1}^{\alpha}$, a stable random variable with characteristic function $e^{-C(\alpha)|u|^{\alpha}}$ (see [9] assuming that $a$ is constant and $\left(L_{t}\right)$ is a truncated $\alpha$-stable process or [18] for more general assumptions on the coefficients assuming that $\left(L_{t}\right)$ is an $\alpha$-stable process). Note that $\frac{a\left(x_{0}, \sigma\right)}{n^{1 / \alpha}} p_{1 / n}\left(x_{0}, \frac{a\left(x_{0}, \sigma\right)}{n^{1 / \alpha}} y+\xi_{1 / n}^{x_{0}}(\theta)\right)$ is the density of $n^{1 / \alpha}\left(X_{1 / n}-\xi_{1 / n}^{x_{0}}(\theta)\right) / a\left(x_{0}, \sigma\right)$ and that we also give in Section 4 a rate of convergence in total variation distance between the law of $n^{1 / \alpha}\left(X_{1 / n}-\xi_{1 / n}^{x_{0}}(\theta)\right) / a\left(x_{0}, \sigma\right)$ and the law of $L_{1}^{\alpha}$.

This observation suggests to approximate $p_{1 / n}(x, y)$ by $\frac{n^{1 / \alpha}}{a(x, \sigma)} \varphi_{\alpha}\left(n^{1 / \alpha} \frac{\left(y-\xi_{1 / n}^{x}(\theta)\right)}{a(x, \sigma)}\right)$ and then approximating the score function, a natural choice of estimating functions is

$$
\begin{array}{r}
g^{1}(x, y, \theta, \sigma)=\frac{n^{1 / \alpha}}{n} \frac{\dot{b}(x, \theta)}{a(x, \sigma)} \frac{\varphi_{\alpha}^{\prime}}{\varphi_{\alpha}}\left(z_{n}(x, y, \theta, \sigma)\right), \\
g^{2}(x, y, \theta, \sigma)=\frac{\dot{a}(x, \sigma)}{a(x, \sigma)}\left(1+z_{n}(x, y, \theta, \sigma) \frac{\varphi_{\alpha}^{\prime}}{\varphi_{\alpha}}\left(z_{n}(x, y, \theta, \sigma)\right)\right),
\end{array}
$$

where

$$
z_{n}(x, y, \theta, \sigma)=n^{1 / \alpha} \frac{\left(y-\xi_{1 / n}^{x}(\theta)\right)}{a(x, \sigma)} .
$$

For this choice of estimating functions, we prove below that solving $G_{n}(\theta, \sigma)=0$ gives a consistent and asymptotic mixed normal estimator of $\left(\theta_{0}, \sigma_{0}\right)$. This result is based on limit theorems for normalized sums

$$
\frac{1}{n} \sum_{i=1}^{n} f\left(X_{\frac{i-1}{n}}, \theta, \sigma\right) h\left(z_{n}\left(X_{\frac{i-1}{n}}, X_{\frac{i}{n}}, \theta, \sigma\right)\right)
$$

where $z_{n}$ is defined by (3.4). We first establish a uniform Law of Large Numbers for $(\theta, \sigma)$ in $V_{n}^{(\eta)}\left(\theta_{0}, \sigma_{0}\right)$, where for $\eta>0$

$$
V_{n}^{(\eta)}\left(\theta_{0}, \sigma_{0}\right)=\left\{(\theta, \sigma) ;\left\|u_{n}^{-1}\left(\theta-\theta_{0}, \sigma-\sigma_{0}\right)^{T}\right\| \leq \eta\right\},
$$

with

$$
u_{n}=\left(\begin{array}{ll}
\frac{1}{n^{1 / \alpha-1 / 2}} & 0 \\
0 & \frac{1}{n^{1 / 2}}
\end{array}\right) .
$$


Theorem 3.1. We assume that $H 1$ and H2 hold. Let $h: \mathbb{R} \mapsto \mathbb{R}$ be a continuous bounded function with bounded derivative and let $f: \mathbb{R} \times \mathbb{R}^{2} \mapsto \mathbb{R}$ be a continuous function with continuous partial derivative with respect to the second variable such that

$$
\sup _{(\theta, \sigma) \in V_{\theta_{0}} \times V_{\sigma_{0}}}\left(|f(x, \theta, \sigma)|+\left|\partial_{\theta} f(x, \theta, \sigma)\right|+\left|\partial_{\sigma} f(x, \theta, \sigma)\right|\right) \leq C\left(1+|x|^{p}\right), \quad \text { for some } p>0 .
$$

Then we have the convergence in probability:

i)

$$
\sup _{(\theta, \sigma) \in V_{n}^{(\eta)}\left(\theta_{0}, \sigma_{0}\right)}\left|\frac{1}{n} \sum_{i=1}^{n} f\left(X_{\frac{i-1}{n}}, \theta, \sigma\right) h\left(z_{n}\left(X_{\frac{i-1}{n}}, X_{\frac{i}{n}}, \theta, \sigma\right)\right)-\int_{0}^{1} f\left(X_{s}, \theta_{0}, \sigma_{0}\right) d s \mathbb{E} h\left(L_{1}^{\alpha}\right)\right| \rightarrow 0 .
$$

ii) Moreover if $\mathbb{E} h\left(L_{1}^{\alpha}\right)=0$

$$
\sup _{(\theta, \sigma) \in V_{n}^{(\eta)}\left(\theta_{0}, \sigma_{0}\right)}\left|\frac{1}{n^{1 / \alpha}} \sum_{i=1}^{n} f\left(X_{\frac{i-1}{n}}, \theta, \sigma\right) h\left(z_{n}\left(X_{\frac{i-1}{n}}, X_{\frac{i}{n}}, \theta, \sigma\right)\right)\right| \rightarrow 0 \quad \text { in probability. }
$$

Obviously ii) is a consequence of i) in the case $\alpha \leq 1$ and only the case $\alpha>1$ requires a proof.

We also establish the stable convergence in law of functionals of the form

$$
\frac{1}{n^{1 / 2}} \sum_{i=1}^{n} f\left(X_{\frac{i-1}{n}}, \theta_{0}, \sigma_{0}\right) h\left(z_{n}\left(X_{\frac{i-1}{n}}, X_{\frac{i}{n}}, \theta_{0}, \sigma_{0}\right)\right) .
$$

Thanks to the control in total variation distance given in Section 4 between $z_{n}\left(X_{\frac{i-1}{n}}, X_{\frac{i}{n}}, \theta_{0}, \sigma_{0}\right)$ and $n^{1 / \alpha} \Delta L_{i}$, this can be reduced to the stable convergence in law for

$$
\frac{1}{n^{1 / 2}} \sum_{i=1}^{n} f\left(X_{\frac{i-1}{n}}, \theta_{0}, \sigma_{0}\right) h\left(n^{1 / \alpha} \Delta L_{i}\right)
$$

This is established in the next theorem.

Theorem 3.2. We assume that $H 1$ and $H 2$ hold. Let $h_{1}, h_{2}: \mathbb{R} \rightarrow \mathbb{R}$ be bounded functions and let $f_{1}, f_{2}$ : $\mathbb{R} \rightarrow \mathbb{R}$ be continuous functions. We assume that $\mathbb{E} h_{1}\left(L_{1}^{\alpha}\right)=\mathbb{E} h_{2}\left(L_{1}^{\alpha}\right)=\mathbb{E} h_{1}\left(L_{1}^{\alpha}\right) h_{2}\left(L_{1}^{\alpha}\right)=0$. Then we have the stable convergence in law with respect to $\sigma\left(L_{s}, s \leq 1\right)$ :

$$
\frac{1}{n^{1 / 2}} \sum_{i=1}^{n}\left(\begin{array}{c}
f_{1}\left(X_{\frac{i-1}{n}}\right) h_{1}\left(n^{1 / \alpha} \Delta L_{i}\right) \\
f_{2}\left(X_{\frac{i-1}{n}}\right) h_{2}\left(n^{1 / \alpha} \Delta L_{i}\right)
\end{array}\right) \Longrightarrow \Sigma^{1 / 2} \mathcal{N}
$$

where $\mathcal{N}$ is a standard Gaussian variable independent of $\Sigma$ and

$$
\Sigma=\left(\begin{array}{cc}
\int_{0}^{1} f_{1}^{2}\left(X_{s}\right) d s \mathbb{E} h_{1}^{2}\left(L_{1}^{\alpha}\right) & 0 \\
0 & \int_{0}^{1} f_{2}^{2}\left(X_{s}\right) d s \mathbb{E} h_{2}^{2}\left(L_{1}^{\alpha}\right)
\end{array}\right) .
$$

From this theorem we deduce the following corollary.

Corollary 3.1. We assume that $H 1$ and H2 hold. Let $h_{1}, h_{2}: \mathbb{R} \rightarrow \mathbb{R}$ be bounded functions with bounded derivative and let $f_{1}, f_{2}: \mathbb{R} \rightarrow \mathbb{R}$ be continuous functions. We assume that $\mathbb{E} h_{1}\left(L_{1}^{\alpha}\right)=\mathbb{E} h_{2}\left(L_{1}^{\alpha}\right)=$ $\mathbb{E} h_{1}\left(L_{1}^{\alpha}\right) h_{2}\left(L_{1}^{\alpha}\right)=0$. Then we have the stable convergence in law with respect to $\sigma\left(L_{s}, s \leq 1\right)$ :

$$
\frac{1}{n^{1 / 2}} \sum_{i=1}^{n}\left(\begin{array}{c}
f_{1}\left(X_{\frac{i-1}{n}}\right) h_{1}\left(z_{n}\left(X_{\frac{i-1}{n}}, X_{\frac{i}{n}}, \theta_{0}, \sigma_{0}\right)\right) \\
f_{2}\left(X_{\frac{i-1}{n}}\right) h_{2}\left(z_{n}\left(X_{\frac{i-1}{n}}, X_{\frac{i}{n}}, \theta_{0}, \sigma_{0}\right)\right)
\end{array}\right) \Longrightarrow \Sigma^{1 / 2} \mathcal{N},
$$

where $\mathcal{N}$ is a standard Gaussian variable independent of $\Sigma$ and

$$
\Sigma=\left(\begin{array}{cc}
\int_{0}^{1} f_{1}^{2}\left(X_{s}\right) d s \mathbb{E} h_{1}^{2}\left(L_{1}^{\alpha}\right) & 0 \\
0 & \int_{0}^{1} f_{2}^{2}\left(X_{s}\right) d s \mathbb{E} h_{2}^{2}\left(L_{1}^{\alpha}\right)
\end{array}\right) .
$$


The proofs of Theorem 3.1, Theorem 3.2 and Corollary 3.1 are postponed to Section 5. They are mainly based on the total variation distance estimates given in Section 4.

From these results, we can establish the asymptotic properties of our estimating functions based estimator.

Theorem 3.3. Let $G_{n}$ be defined by (3.1) with $g^{1}$ and $g^{2}$ given by (3.2) and (3.3). Under the assumptions H1, H2 and H3, there exists an estimator $\left(\hat{\theta}_{n}, \hat{\sigma}_{n}\right)$ solving the equation $G_{n}(\theta, \sigma)=0$ with probability tending to 1 , that converges in probability to $\left(\theta_{0}, \sigma_{0}\right)$. Moreover we have the convergence in law

$$
\left(\begin{array}{c}
n^{1 / \alpha-1 / 2}\left(\hat{\theta}_{n}-\theta_{0}\right) \\
n^{1 / 2}\left(\hat{\sigma}_{n}-\sigma_{0}\right)
\end{array}\right) \Longrightarrow I\left(\theta_{0}, \sigma_{0}\right)^{-1 / 2} \mathcal{N}
$$

where $\mathcal{N}$ is a standard Gaussian variable independent of $I\left(\theta_{0}, \sigma_{0}\right)$ and

$$
I(\theta, \sigma)=\left(\begin{array}{cc}
\int_{0}^{1} \frac{\dot{b}\left(X_{s}, \theta\right)^{2}}{a\left(X_{s}, \sigma\right)^{2}} d s \int_{\mathbb{R}} \frac{\varphi_{\alpha}^{\prime}(u)^{2}}{\varphi_{\alpha}(u)} d u & 0 \\
0 & \int_{0}^{1} \frac{\dot{a}\left(X_{s}, \sigma\right)^{2}}{a\left(X_{s}, \sigma\right)^{2}} d s \int_{\mathbb{R}} \frac{\left(\varphi_{\alpha}(u)+u \varphi_{\alpha}^{\prime}(u)\right)^{2}}{\varphi_{\alpha}(u)} d u
\end{array}\right) .
$$

Before proving Theorem 3.3, we make some remarks.

Remark 3.1. We observe that the estimation rate for the drift parameter $\theta$ degenerates as a goes to 2 . Indeed, the limit case $\alpha=2$ corresponds to the situation where $\left(L_{t}\right)$ is a Brownian motion and where the estimation of $\theta$ is impossible from high frequency observations on a fixed time period. On the contrary, when $\alpha<1$, the estimation rate for $\theta$ is faster than the usual one $n^{1 / 2}$.

Remark 3.2. The LAMN property with information $I(\theta, \sigma)$ has been established in [8] for this experiment, assuming that a is constant and $g$ is a truncation function. This result permits to deduce that our estimator is efficient.

If the solution to the ordinary differential equation (2.2) is not explicit, we can replace it in the expression of $z_{n}$ by a numerical scheme $\bar{\xi}_{1 / n}^{x}(\theta)$. The next proposition gives sufficient conditions on the scheme to preserve the conclusion of Theorem 3.3.

Proposition 3.1. Let $\bar{\xi}_{1 / n}^{x}(\theta)$ be an approximation of $\xi_{1 / n}^{x}(\theta)$ such that

i) $\sup _{\theta \in V_{\theta_{0}}}\left|\xi_{1 / n}^{x}(\theta)-\bar{\xi}_{1 / n}^{x}(\theta)\right| \leq C\left(1+|x|^{p}\right) / n^{k}$ with $k>1 / \alpha+1 / 2$,

ii) $\sup _{\theta \in V_{\theta_{0}}}\left|\partial_{\theta} \bar{\xi}_{1 / n}^{x}(\theta)-\frac{\dot{b}(x, \theta)}{n}\right| \leq C\left(1+|x|^{p}\right) / n^{2}$, for some $p>0$.

Then under H1, H2 and H3 if we replace $z_{n}$ by $\bar{z}_{n}(x, y, \theta, \sigma)=n^{1 / \alpha} \frac{\left(y-\bar{\xi}_{1 / n}^{x}(\theta)\right)}{a(x, \sigma)}$ in equations (3.2) and (3.3), there exists an estimator $\left(\bar{\theta}_{n}, \bar{\sigma}_{n}\right)$ that solves $G_{n}(\theta, \sigma)=0$ with probability tending to 1 . This estimator has the same asymptotic properties as the estimator defined in Theorem 3.3.

The proof of Proposition 3.1 is omitted since it follows the same arguments as the proof of Theorem 3.3 replacing $z_{n}$ by $\bar{z}_{n}$.

Example 3.1. We assume that the function $b$ is of class $\mathcal{C}^{k}$ with $k \geq 2$ and that

$$
\sup _{\theta \in V_{\theta_{0}}} \max _{1 \leq l \leq k-1}\left(\left|\partial_{x}^{l} b(x, \theta)\right|+\left|\partial_{\theta} \partial_{x}^{l} b(x, \theta)\right|\right) \leq C\left(1+|x|^{p}\right) .
$$

For $f: \mathbb{R} \times V_{\theta_{0}} \mapsto \mathbb{R}$ of class $\mathcal{C}^{k}$, we set $A^{0} f=f, A f=b f^{\prime}$ and define recursively $A^{j} f=A\left(A^{j-1} f\right)$ for $2 \leq j \leq k$. We have $f\left(\xi_{t}^{x}(\theta), \theta\right)=f(x, \theta)+\int_{0}^{t}(A f)\left(\xi_{s}^{x}(\theta), \theta\right) d s$ and consequently we obtain

$$
\begin{aligned}
\xi_{t}^{x}(\theta) & =x+\int_{0}^{t}\left(A^{0} b\right)\left(\xi_{s}^{x}(\theta), \theta\right) d s \\
& =x+t\left(A^{0} b\right)(x, \theta)+\int_{0}^{t} \int_{0}^{t_{1}}(A b)\left(\xi_{t_{2}}^{x}(\theta), \theta\right) d t_{2} d t_{1} \\
& =\bar{\xi}_{t}^{x}(\theta)+\int_{0}^{t} \int_{0}^{t_{1}} \ldots \int_{0}^{t_{k-1}}\left(A^{k-1} b\right)\left(\xi_{t_{k}}^{x}(\theta), \theta\right) d t_{k} \ldots d t_{t_{1}}
\end{aligned}
$$


with $\bar{\xi}_{t}^{x}(\theta)=x+t\left(A^{0} b\right)(x, \theta)+\frac{t^{2}}{2}(A b)(x, \theta)+\ldots+\frac{t^{k-1}}{(k-1) !}\left(A^{k-2} b\right)(x, \theta)$. We deduce that if $k>1 / \alpha+1 / 2$, i) and ii) are satisfied and the result of Proposition 3.1 can be applied. If $\alpha>2 / 3$, the simple choice $\bar{\xi}_{1 / n}^{x}(\theta)=x+b(x, \theta) / n$ is convenient.

Proof of Theorem 3.3. The proof is based on the results established in Sørensen [23] to obtain the consistency and asymptotic normality of estimators constructed from estimating functions. We first remark that from H3, we have $I\left(\theta_{0}, \sigma_{0}\right)>0$ almost surely.

We define the matrix $J_{n}\left(\left(\theta_{1}, \sigma_{1}\right),\left(\theta_{2}, \sigma_{2}\right)\right)$ by

$$
J_{n}\left(\left(\theta_{1}, \sigma_{1}\right),\left(\theta_{2}, \sigma_{2}\right)\right)=\sum_{i=1}^{n}\left(\begin{array}{cc}
\partial_{\theta} g^{1}\left(X_{\frac{i-1}{n}}, X_{\frac{i}{n}}, \theta_{1}, \sigma_{1}\right) & \partial_{\sigma} g^{1}\left(X_{\frac{i-1}{n}}, X_{\frac{i}{n}}, \theta_{1}, \sigma_{1}\right) \\
\partial_{\theta} g^{2}\left(X_{\frac{i-1}{n}}, X_{\frac{i}{n}}, \theta_{2}, \sigma_{2}\right) & \partial_{\sigma} g^{2}\left(X_{\frac{i-1}{n}}, X_{\frac{i}{n}}, \theta_{2}, \sigma_{2}\right)
\end{array}\right)
$$

We also recall that $V_{n}^{(\eta)}\left(\theta_{0}, \sigma_{0}\right)$ and $u_{n}$ are respectively defined by (3.5) and (3.6). With these notations, the result of Theorem 3.3 is a consequence of the two following sufficient conditions:

C1 : $\forall \eta>0$, we have the convergence in probability :

$$
\sup _{\left(\theta_{1}, \sigma_{1}\right),\left(\theta_{2}, \sigma_{2}\right) \in V_{n}^{(\eta)}\left(\theta_{0}, \sigma_{0}\right)}\left\|u_{n} J_{n}\left(\left(\theta_{1}, \sigma_{1}\right),\left(\theta_{2}, \sigma_{2}\right)\right) u_{n}-I\left(\theta_{0}, \sigma_{0}\right)\right\| \rightarrow 0 .
$$

C2 : $\left(u_{n} G_{n}\left(\theta_{0}, \sigma_{0}\right)\right)_{n}$ stably converges in law to $I\left(\theta_{0}, \sigma_{0}\right)^{1 / 2} \mathcal{N}$, where $\mathcal{N}$ is a standard Gaussian variable independent of $I\left(\theta_{0}, \sigma_{0}\right)$ and the convergence is stable with respect to the $\sigma$-field $\sigma\left(L_{s}, s \leq 1\right)$.

These conditions imply the ones given in [23] which are sufficient to prove consistency in the case of a random information matrix. In [23] the Central Limit Theorem is established for a deterministic information only, however the proof can be easily extended to the random case enhancing the convergence in law by the stable convergence in law.

To check the conditions $\mathbf{C 1}$ and $\mathbf{C 2}$, we first recall that

$$
u_{n} J_{n}\left(\left(\theta_{1}, \sigma_{1}\right),\left(\theta_{2}, \sigma_{2}\right)\right) u_{n}=\sum_{i=1}^{n}\left(\begin{array}{cc}
\frac{n}{n^{2 / \alpha}} \partial_{\theta} g^{1}\left(X_{\frac{i-1}{n}}, X_{\frac{i}{n}}, \theta_{1}, \sigma_{1}\right) & \frac{1}{n^{1 / \alpha}} \partial_{\sigma} g^{1}\left(X_{\frac{i-1}{n}}, X_{\frac{i}{n}}, \theta_{1}, \sigma_{1}\right) \\
\frac{1}{n^{1 / \alpha}} \partial_{\theta} g^{2}\left(X_{\frac{i-1}{n}}, X_{\frac{i}{n}}, \theta_{2}, \sigma_{2}\right) & \frac{1}{n} \partial_{\sigma} g^{2}\left(X_{\frac{i-1}{n}}, X_{\frac{i}{n}}, \theta_{2}, \sigma_{2}\right)
\end{array}\right)
$$

We compute explicitly the partial derivatives appearing in this matrix. Recalling that $g^{1}$ and $g^{2}$ are given by (3.2) and (3.3) and observing that $\partial_{\theta} z_{n}(x, y, \theta, \sigma)=-\frac{n^{1 / \alpha}}{a(x, \sigma)} \dot{\xi}_{1 / n}^{x}(\theta)$ and $\partial_{\sigma} z_{n}(x, y, \theta, \sigma)=-\frac{\dot{a}(x, \sigma)}{a(x, \sigma)} z_{n}(x, y, \theta, \sigma)$ we get

$$
\begin{gathered}
\partial_{\theta} g^{1}(x, y, \theta, \sigma)=\frac{n^{1 / \alpha}}{n} \frac{\ddot{b}(x, \theta)}{a(x, \sigma)} \frac{\varphi_{\alpha}^{\prime}}{\varphi_{\alpha}}\left(z_{n}(x, y, \theta, \sigma)\right)-\frac{n^{2 / \alpha}}{n} \frac{\dot{b}(x, \theta)}{a(x, \sigma)^{2}} \dot{\xi}_{1 / n}^{x}(\theta)\left(\frac{\varphi_{\alpha}^{\prime}}{\varphi_{\alpha}}\right)^{\prime}\left(z_{n}(x, y, \theta, \sigma)\right), \\
\partial_{\sigma} g^{1}(x, y, \theta, \sigma)=-\frac{n^{1 / \alpha}}{n} \frac{\dot{a}(x, \sigma)}{a(x, \sigma)^{2}} \dot{b}(x, \theta)\left[\frac{\varphi_{\alpha}^{\prime}}{\varphi_{\alpha}}\left(z_{n}(x, y, \theta, \sigma)\right)+z_{n}(x, y, \theta, \sigma)\left(\frac{\varphi_{\alpha}^{\prime}}{\varphi_{\alpha}}\right)^{\prime}\left(z_{n}(x, y, \theta, \sigma)\right)\right], \\
\partial_{\theta} g^{2}(x, y, \theta, \sigma)=-n^{1 / \alpha} \frac{\dot{a}(x, \sigma)}{a(x, \sigma)^{2}} \dot{\xi}_{1 / n}^{x}(\theta)\left[\frac{\varphi_{\alpha}^{\prime}}{\varphi_{\alpha}}\left(z_{n}(x, y, \theta, \sigma)\right)+z_{n}(x, y, \theta, \sigma)\left(\frac{\varphi_{\alpha}^{\prime}}{\varphi_{\alpha}}\right)^{\prime}\left(z_{n}(x, y, \theta, \sigma)\right)\right], \\
\partial_{\sigma} g^{2}(x, y, \theta, \sigma)=\left(\frac{\ddot{a} a-\dot{a})^{2}}{a^{2}}\right)(x, \sigma)\left[1+z_{n}(x, y, \theta, \sigma) \frac{\varphi_{\alpha}^{\prime}}{\varphi_{\alpha}}\left(z_{n}(x, y, \theta, \sigma)\right]\right. \\
-\left(\frac{\dot{a}^{2}}{a^{2}}\right)(x, \sigma) z_{n}(x, y, \theta, \sigma)\left[\frac{\varphi_{\alpha}^{\prime}}{\varphi_{\alpha}}\left(z_{n}(x, y, \theta, \sigma)\right)+z_{n}(x, y, \theta, \sigma)\left(\frac{\varphi_{\alpha}^{\prime}}{\varphi_{\alpha}}\right)^{\prime}\left(z_{n}(x, y, \theta, \sigma)\right)\right] .
\end{gathered}
$$

\section{Proof of C1.}

We will use intensively the result of Theorem 3.1. 
We first remark that the condition $\mathbf{C} 1$ reduces to the uniform convergence of $u_{n} J_{n}((\theta, \sigma),(\theta, \sigma)) u_{n}$. In the sequel we use the notation $h_{\alpha}=\frac{\varphi_{\alpha}^{\prime}}{\varphi_{\alpha}}$ and $g_{\alpha}(z)=h_{\alpha}(z)+z h_{\alpha}^{\prime}(z)$. The functions $h_{\alpha}$ and $g_{\alpha}$ are bounded with bounded derivative (see [2]). We also set :

$$
u_{n} J_{n}((\theta, \sigma),(\theta, \sigma)) u_{n}=\left(\begin{array}{cc}
I_{n}^{1,1} & I_{n}^{1,2} \\
I_{n}^{2,1} & I_{n}^{2,2}
\end{array}\right) .
$$

With these notations we have using the above calculus

$$
\begin{gathered}
I_{n}^{1,1}=\frac{1}{n^{1 / \alpha}} \sum_{i=1}^{n} \frac{\ddot{b}\left(X_{\frac{i-1}{n}}, \theta\right)}{a\left(X_{\frac{i-1}{n}}, \sigma\right)} h_{\alpha}\left(z_{n}\left(X_{\frac{i-1}{n}}, X_{\frac{i}{n}}, \theta, \sigma\right)\right)-\sum_{i=1}^{n} \frac{\dot{b}\left(X_{\frac{i-1}{n}}, \theta\right)}{a\left(X_{\frac{i-1}{n}}, \sigma\right)^{2}} \dot{\xi}_{1 / n} X_{\frac{i-1}{n}}(\theta) h_{\alpha}^{\prime}\left(z_{n}\left(X_{\frac{i-1}{n}}, X_{\frac{i}{n}}, \theta, \sigma\right)\right), \\
I_{n}^{1,2}=-\frac{1}{n} \sum_{i=1}^{n} \frac{\dot{a}\left(X_{\frac{i-1}{n}}, \sigma\right)}{a\left(X_{\frac{i-1}{n}}, \sigma\right)^{2}} \dot{b}\left(X_{\frac{i-1}{n}}, \theta\right) g_{\alpha}\left(z_{n}\left(X_{\frac{i-1}{n}}, X_{\frac{i}{n}}, \theta, \sigma\right)\right), \\
I_{n}^{2,1}=-\sum_{i=1}^{n} \frac{\dot{a}\left(X_{\frac{i-1}{n}}, \sigma\right)}{a\left(X_{\frac{i-1}{n}}, \sigma\right)^{2}} \dot{\xi}_{1 / n} X_{\frac{i-1}{n}}(\theta) g_{\alpha}\left(z_{n}\left(X_{\frac{i-1}{n}}, X_{\frac{i}{n}}, \theta, \sigma\right)\right), \\
I_{n}^{2,2}=\frac{1}{n} \sum_{i=1}^{n}\left(\frac{\ddot{a} a-\dot{a}^{2}}{a^{2}}\right)\left(X_{\frac{i-1}{n}}, \sigma\right)\left[1+z_{n}\left(X_{\frac{i-1}{n}}, X_{\frac{i}{n}}, \theta, \sigma\right) h_{\alpha}\left(z_{n}\left(X_{\frac{i-1}{n}}, X_{\frac{i}{n}}, \theta, \sigma\right)\right]\right. \\
-\frac{1}{n} \sum_{i=1}^{n}\left(\frac{\dot{a}^{2}}{a^{2}}\right)\left(X_{\frac{i-1}{n}}, \sigma\right) z_{n}\left(X_{\frac{i-1}{n}}, X_{\frac{i}{n}}, \theta, \sigma\right) g_{\alpha}\left(z_{n}\left(X_{\frac{i-1}{n}}, X_{\frac{i}{n}}, \theta, \sigma\right)\right) .
\end{gathered}
$$

From H1, obviously the functions $\dot{b} / a, \dot{a} / a, \ddot{b} / a, \ddot{a} / a$ satisfy the assumptions of Theorem 3.1.

Since $\mathbb{E} h_{\alpha}\left(L_{1}^{\alpha}\right)=0$, the first term in the expression of $I_{n}^{1,1}$ goes to zero (Theorem $3.1 \mathrm{ii}$ )).

For the second term we observe that $\left(\dot{\xi}_{t}^{x}(\theta)\right)_{t}$ solves

$$
\dot{\xi}_{t}^{x}(\theta)=\int_{0}^{t} b^{\prime}\left(\xi_{s}^{x}(\theta), \theta\right) \dot{\xi}_{s}^{x}(\theta) d s+\int_{0}^{t} \dot{b}\left(\xi_{s}^{x}(\theta), \theta\right) d s,
$$

and from $\mathrm{H} 1$ and Gronwall's Lemma we can deduce (we omit the details of this standard proof)

$$
\sup _{\theta \in V_{\theta_{0}}}\left|\dot{\xi}_{1 / n}^{x}(\theta)-\frac{1}{n} \dot{b}(x, \theta)\right| \leq C\left(1+|x|^{p}\right) / n^{2}
$$

so from Theorem $3.1 \mathrm{i})$ we deduce the convergence of the second term of $I_{n}^{1,1}$ to $-\int_{0}^{1} \frac{\dot{b}\left(X_{s}, \theta_{0}\right)^{2}}{a\left(X_{s}, \sigma_{0}\right)^{2}} d s \mathbb{E}\left(\frac{\varphi_{\alpha}^{\prime}}{\varphi_{\alpha}}\right)^{\prime}\left(L_{1}^{\alpha}\right)$. Since $\int \varphi_{\alpha}^{\prime \prime}(x) d x=0$, we deduce $\mathbb{E}\left(\frac{\varphi_{\alpha}^{\prime}}{\varphi_{\alpha}}\right)^{\prime}\left(L_{1}^{\alpha}\right)=-\mathbb{E}\left(\frac{\varphi_{\alpha}^{\prime}}{\varphi_{\alpha}}\right)^{2}\left(L_{1}^{\alpha}\right)$ and finally

$$
\sup _{(\theta, \sigma) \in V_{n}^{(\eta)}\left(\theta_{0}, \sigma_{0}\right)}\left|I_{n}^{1,1}-\int_{0}^{1} \frac{\dot{b}\left(X_{s}, \theta_{0}\right)^{2}}{a\left(X_{s}, \sigma_{0}\right)^{2}} d s \mathbb{E}\left(\frac{\varphi_{\alpha}^{\prime}}{\varphi_{\alpha}}\right)^{2}\left(L_{1}^{\alpha}\right)\right| \rightarrow 0 .
$$

Using the symmetry of the function $\varphi_{\alpha}$ we have $\mathbb{E} g_{\alpha}\left(L_{1}^{\alpha}\right)=0$ and we deduce easily from Theorem 3.1 i) and (3.7) that

$$
\sup _{(\theta, \sigma) \in V_{n}^{(\eta)}\left(\theta_{0}, \sigma_{0}\right)}\left|I_{n}^{1,2}\right| \rightarrow 0 \quad \text { and } \quad \sup _{(\theta, \sigma) \in V_{n}^{(\eta)}\left(\theta_{0}, \sigma_{0}\right)}\left|I_{n}^{2,1}\right| \rightarrow 0 .
$$

Turning to $I_{n}^{2,2}$, we have by integrating by part $\mathbb{E}\left(1+L_{1}^{\alpha} h_{\alpha}\left(L_{1}^{\alpha}\right)\right)=0$. Consequently

$$
\sup _{(\theta, \sigma) \in V_{n}^{(\eta)}\left(\theta_{0}, \sigma_{0}\right)}\left|I_{n}^{2,2}+\int_{0}^{1}\left(\frac{\dot{a}^{2}}{a^{2}}\right)\left(X_{s}, \sigma_{0}\right) d s \mathbb{E} L_{1}^{\alpha} g_{\alpha}\left(L_{1}^{\alpha}\right)\right| \rightarrow 0,
$$


and it remains to check $\mathbb{E} L_{1}^{\alpha} g_{\alpha}\left(L_{1}^{\alpha}\right)=-\mathbb{E} \frac{\left(\varphi_{\alpha}\left(L_{1}^{\alpha}\right)+L_{1}^{\alpha} \varphi_{\alpha}^{\prime}\left(L_{1}^{\alpha}\right)\right)^{2}}{\varphi_{\alpha}\left(L_{1}^{\alpha}\right)^{2}}$. This is done by integrating by parts. This achieves the proof of $\mathbf{C 1}$.

\section{Proof of C2.}

We recall that

$$
u_{n} G_{n}\left(\theta_{0}, \sigma_{0}\right)=\frac{1}{n^{1 / 2}} \sum_{i=1}^{n}\left(\begin{array}{c}
\frac{\dot{b}\left(X_{\frac{i-1}{n}}, \theta_{0}\right)}{a\left(X_{\frac{i-1}{n}}, \sigma_{0}\right)} \frac{\varphi_{\alpha}^{\prime}}{\varphi_{\alpha}}\left(z_{n}\left(X_{\frac{i-1}{n}}, X_{\frac{i}{n}}, \theta_{0}, \sigma_{0}\right)\right) \\
\frac{\dot{a}\left(X_{\left.\frac{i-1}{n}, \sigma_{0}\right)}\right.}{a\left(X_{\frac{i-1}{n}}, \sigma_{0}\right)}\left[1+z_{n}\left(X_{\frac{i-1}{n}}, X_{\frac{i}{n}}, \theta_{0}, \sigma_{0}\right) \frac{\varphi_{\alpha}^{\prime}}{\varphi_{\alpha}}\left(z_{n}\left(X_{\frac{i-1}{n}}, X_{\frac{i}{n}}, \theta_{0}, \sigma_{0}\right)\right)\right]
\end{array}\right) .
$$

Applying Corollary 3.1 with $f_{1}=\dot{b} / a, f_{2}=\dot{a} / a, h_{1}=\varphi_{\alpha}^{\prime} / \varphi_{\alpha}, h_{2}(z)=1+z h_{1}(z)\left(h_{1}\right.$ and $h_{2}$ are bounded functions with bounded derivative, see for example [2]), we deduce immediately C2.

\section{Total variation distance estimates}

This section is the most technical part of the paper and contains some crucial estimates to derive the asymptotic properties of the estimating functions considered in the previous section. We consider here the process $\left(X_{t}\right)_{t \in[0,1]}$ that solves $(2.1)$ for the value $\left(\theta_{0}, \sigma_{0}\right)$ of the parameter and to simplify the notation we omit the dependence on $\left(\theta_{0}, \sigma_{0}\right)$ in the expressions of the functions $a, b$ and $\xi^{x_{0}}$.

We will prove that we can approximate $n^{1 / \alpha}\left(X_{\frac{1}{n}}-\xi_{\frac{1}{n}}^{x_{0}}\right)$ by $n^{1 / \alpha} a\left(x_{0}\right) L_{\frac{1}{n}}$ and control this approximation. This is done by estimating the total variation distance between $n^{1 / \alpha}\left(X_{\frac{1}{n}}-\xi_{\frac{1}{n}}^{x_{0}}\right)$ and $n^{1 / \alpha} a\left(x_{0}\right) L_{\frac{1}{n}}$. We also give a weak rate of convergence of the rescaled Lévy process $n^{1 / \alpha} L_{1 / n}$ to the $\alpha$-stable process $L_{1}^{\alpha}$ which is estimated by the total variation distance between $n^{1 / \alpha} L_{1 / n}$ and $L_{1}^{\alpha}$.

Theorem 4.1. We assume H1(a) and H2. There exists a constant $C$ such that for any measurable bounded function $h$, we have :

$$
\left|\mathbb{E} h\left(n^{1 / \alpha}\left(X_{\frac{1}{n}}-\xi_{\frac{1}{n}}^{x_{0}}\right)\right)-\mathbb{E} h\left(n^{1 / \alpha} a\left(x_{0}\right) L_{\frac{1}{n}}\right)\right| \leq C\left(1+\left|x_{0}\right|\right) \varepsilon_{n}\|h\|_{\infty},
$$

where

- if $\alpha \leq 1, \forall \varepsilon \in(0,1), \varepsilon_{n}=\frac{1}{n^{1-\varepsilon}}$

- if $\alpha>1, \forall \varepsilon \in(0,1 / \alpha), \varepsilon_{n} \stackrel{n^{1-\varepsilon}}{=} \frac{1}{n^{1 / \alpha-\varepsilon}}$.

In particular, in both cases $n^{1 / 2} \varepsilon_{n} \rightarrow 0$.

Theorem 4.2. Under H2, we have for any bounded function $h$ :

$$
\left|\mathbb{E} h\left(n^{1 / \alpha} L_{1 / n}\right)-\mathbb{E} h\left(L_{1}^{\alpha}\right)\right| \leq C\|h\|_{\infty} \varepsilon_{n},
$$

where $\varepsilon_{n}$ is as in Theorem 4.1.

Combining Theorem 4.1 and Theorem 4.2, we see that the total variation distance between the distribution of $\frac{n^{1 / \alpha}}{a\left(x_{0}\right)}\left(X_{\frac{1}{n}}-\xi_{\frac{1}{n}}^{x_{0}}\right)$ and the distribution of $L_{1}^{\alpha}$ is bounded by $C\left(1+\left|x_{0}\right|\right) \varepsilon_{n}$.

To prove these results, it is convenient to introduce an adequate truncation function and to consider a rescaled process. This is explained in the next subsections. Moreover, the proof of Theorems 4.1 and 4.2 requires some Malliavin calculus and we recall in what follows all the technical tools to make easier the understanding of the paper.

Remark 4.1. In the statement of Theorem 4.1, we give a control for the distance in total variation between the laws of the processes $X$ and $L$, under a short time asymptotic $1 / n \rightarrow 0$. If we assume that $h$ admits a bounded derivative, it is possible to get some related control from the study of the strong error $\mid n^{1 / \alpha}\left(X_{\frac{1}{n}}-\right.$ 
$\left.\xi_{\frac{1}{n}}^{x_{0}}\right)-n^{1 / \alpha} a\left(x_{0}\right) L_{\frac{1}{n}} \mid$. In Lemma 4.2 below we state an upper bound, in probability, for this error. In the case $\alpha^{n}>1$, using the controls in $L^{p}$-norm given in the proof of Lemma 4.2, we can show

$$
\left|\mathbb{E} h\left(n^{1 / \alpha}\left(X_{\frac{1}{n}}-\xi_{\frac{1}{n}}^{x_{0}}\right)\right)-\mathbb{E} h\left(n^{1 / \alpha} a\left(x_{0}\right) L_{\frac{1}{n}}\right)\right| \leq C\left(1+\left|x_{0}\right|\right)\left(\|h\|_{\infty}+\left\|h^{\prime}\right\|_{\infty}\right) / n^{1 / \alpha-\varepsilon} .
$$

Unfortunately, this proof does not work in the case $\alpha \leq 1$ and we have not been able to give a simple proof of the above result in that case. The Malliavin calculus, especially the integration by part formula and the Malliavin weights, permit to compensate the lack of integrability of the process $\left(L_{t}\right)$ and additionally to weaken the assumptions on the function $h$.

\subsection{Localization and rescaling}

We first introduce a truncation function in order to suppress the big jumps of $\left(L_{t}\right)$. Let $\tau: \mathbb{R} \mapsto[0,1]$ be a symmetric function, continuous with continuous derivative, such that $\tau=1$ on $\{|z| \leq K(a) / 2\}, \tau=0$ on $\{|z| \geq K(a)\}$ where

$$
K(a)=\frac{1}{2}\left(\eta \wedge \frac{1}{\left\|a^{\prime}\right\|_{\infty}}\right)
$$

for $\eta$ defined in $\mathrm{H} 2(\mathrm{~b})$.

On the same probability space $\left(\Omega, \mathcal{F},\left(\mathcal{F}_{t}\right), \mathbb{P}\right)$, we consider the Lévy process $\left(L_{t}\right)_{t \in[0,1]}$ with Lévy measure $\nu$ and the truncated Lévy process $\left(L_{t}^{\tau}\right)_{t \in[0,1]}$ with Lévy measure $\nu^{\tau}$ given by $\nu^{\tau}(d z)=\frac{g(z)}{|z|^{\alpha+1}} \tau(z) 1_{\mathbb{R} \backslash\{0\}}(z) d z$. This can be done by setting $L_{t}=\int_{0}^{t} \int_{\mathbb{R}} z \tilde{\mu}(d s, d z)$, respectively $L_{t}^{\tau}=\int_{0}^{t} \int_{\mathbb{R}} z \tilde{\mu}^{\tau}(d s, d z)$, where $\tilde{\mu}$, respectively $\tilde{\mu}^{\tau}$, are the compensated Poisson random measures associated respectively to

$$
\begin{gathered}
\mu(A)=\int_{[0,1]} \int_{\mathbb{R}} \int_{[0,1]} 1_{A}(t, z) \mu^{g}(d t, d z, d u), \quad A \subset[0,1] \times \mathbb{R} \\
\mu^{\tau}(A)=\int_{[0,1]} \int_{\mathbb{R}} \int_{[0,1]} 1_{A}(t, z) 1_{\{u \leq \tau(z)\}} \mu^{g}(d t, d z, d u), \quad A \subset[0,1] \times \mathbb{R},
\end{gathered}
$$

for $\mu^{g}$ a Poisson random measure on $[0,1] \times \mathbb{R} \times[0,1]$ with compensator $\bar{\mu}^{g}(d t, d z, d u)=d t \frac{g(z)}{|z|^{\alpha+1}} 1_{\mathbb{R} \backslash\{0\}}(z) d z d u$.

By construction, the restrictions of the measures $\mu$ and $\mu^{\tau}$ to $[0,1 / n] \times \mathbb{R}$ coincide on the event

$$
\Omega_{n}=\left\{\omega \in \Omega ; \mu^{g}([0,1 / n] \times\{z \in \mathbb{R} ;|z| \geq K(a) / 2\} \times[0,1])=0\right\} .
$$

Since $\mu^{g}([0,1 / n] \times\{z \in \mathbb{R} ;|z| \geq K(a) / 2\} \times[0,1])$ has a Poisson distribution with parameter

$$
\lambda_{n}=\frac{1}{n} \int_{|z| \geq K(a) / 2} g(z) /|z|^{\alpha+1} d z \leq C / n
$$

we deduce that

$$
\mathbb{P}\left(\Omega_{n}^{c}\right) \leq C / n .
$$

We consider now the truncated process

$$
X_{t}^{\tau}=x_{0}+\int_{0}^{t} b\left(X_{s}^{\tau}\right) d s+\int_{0}^{t} a\left(X_{s-}^{\tau}\right) d L_{s}^{\tau}, \quad t \in[0,1] .
$$

Obviously $\left(X_{t}, L_{t}\right)_{t \in[0,1 / n]}=\left(X_{t}^{\tau}, L_{t}^{\tau}\right)_{t \in[0,1 / n]}$ on $\Omega_{n}$ and consequently since $\mathbb{P}\left(\Omega_{n}^{c}\right) \leq C / n$, the result of Theorem 4.1 consists in proving

$$
\left|\mathbb{E} h\left(n^{1 / \alpha}\left(X_{1 / n}^{\tau}-\xi_{1 / n}^{x_{0}}\right)\right)-\mathbb{E} h\left(n^{1 / \alpha} a\left(x_{0}\right) L_{1 / n}^{\tau}\right)\right| \leq C\left(1+\left|x_{0}\right|\right) \varepsilon_{n}\|h\|_{\infty} .
$$


To clarify the proofs, it will be useful to rescale the truncated process $\left(X_{t}^{\tau}\right)_{t \in[0,1 / n]}$. To this end we introduce an auxiliary Lévy process $\left(L_{t}^{n}\right)_{t \in[0,1]}$ defined possibly on an other filtered space $\left(\bar{\Omega}, \overline{\mathcal{F}},\left(\overline{\mathcal{F}}_{t}\right), \overline{\mathbb{P}}\right)$ and admitting the decomposition

$$
L_{t}^{n}=\int_{0}^{t} \int_{\mathbb{R}} z \tilde{\mu}^{n}(d t, d z), \quad t \in[0,1]
$$

where $\tilde{\mu}^{n}$ is a compensated Poisson random measure, $\tilde{\mu}^{n}=\mu^{n}-\bar{\mu}^{n}$, with compensator $\bar{\mu}^{n}(d t, d z)=$ $d t \frac{g\left(z / n^{1 / \alpha}\right)}{|z|^{\alpha+1}} \tau\left(z / n^{1 / \alpha}\right) 1_{\mathbb{R} \backslash\{0\}}(z) d z$. By construction, the process $\left(L_{t}^{n}\right)_{t \in[0,1]}$ is equal in law to the rescaled truncated process $\left(n^{1 / \alpha} L_{t / n}^{\tau}\right)_{t \in[0,1]}$. We now consider the rescaled stochastic differential equation

$$
Y_{t}^{n}=x_{0}+\frac{1}{n} \int_{0}^{t} b\left(Y_{s}^{n}\right) d s+\frac{1}{n^{1 / \alpha}} \int_{0}^{t} a\left(Y_{s-}^{n}\right) d L_{s}^{n}, \quad t \in[0,1],
$$

and the rescaled ordinary differential equation

$$
\xi_{t}^{n, x_{0}}=x_{0}+\frac{1}{n} \int_{0}^{t} b\left(\xi_{s}^{n, x_{0}}\right) d s, \quad t \in[0,1] .
$$

The equality in law

$$
\left(Y_{t}^{n}-\xi_{t}^{n, x_{0}}, L_{t}^{n}\right)_{t \in[0,1]} \stackrel{\text { law }}{=}\left(X_{t / n}^{\tau}-\xi_{t / n}^{x_{0}}, n^{1 / \alpha} L_{t / n}^{\tau}\right)_{t \in[0,1]}
$$

is straightforward and consequently with these notations the result of Theorem 4.1 follows from

$$
\left|\mathbb{E} h\left(n^{1 / \alpha}\left(Y_{1}^{n}-\xi_{1}^{n, x_{0}}\right)\right)-\mathbb{E} h\left(a\left(x_{0}\right) L_{1}^{n}\right)\right| \leq C\left(1+\left|x_{0}\right|\right) \varepsilon_{n}\|h\|_{\infty} .
$$

It is worth to note that the jumps of $\left(L_{t}^{n}\right)$ are bounded by $n^{1 / \alpha} K(a)$, and then the processes $\left(L_{t}^{n}\right)$ and $\left(Y_{t}^{n}\right)$ admit moments of all orders. More precisely, we have the following result.

Lemma 4.1. Assuming H1(a) and H2(a), we have

$$
\begin{aligned}
& \forall p \geq 1, \quad \sup _{n} \mathbb{E}\left(\sup _{t \in[0,1]}\left|Y_{t}^{n}\right|^{p}\right) \leq C_{p}\left(1+\left|x_{0}\right|^{p}\right), \\
& \forall p \geq 1 \text { and } p>\alpha, \quad \mathbb{E} \sup _{t \in[0,1]}\left|Y_{t}^{n}-x_{0}\right|^{p} \leq C_{p}\left(1+\left|x_{0}\right|^{p}\right) \frac{1}{n} .
\end{aligned}
$$

The proof of both inequalities is based on Burkholder type inequalities (see 2.1.36 and 2.1.37 in Lemma 2.1.5 of [13]) for purely discontinuous martingales and standard arguments (convexity inequality, Lipschitz assumption on the coefficients and Gronwall's lemma). We omit it.

We end this subsection with a control of $\sup _{t \leq 1 / n}\left|n^{1 / \alpha}\left(X_{t}-\xi_{t}^{x_{0}}\left(\theta_{0}\right)\right)-n^{1 / \alpha} a\left(x_{0}\right) L_{t}\right|$ which can be established using both the truncation and rescaling procedure.

Lemma 4.2. Assuming H1(a) and H2(a), there exists $p>0$ such that for any $\varepsilon>0$

$$
\mathbb{P}\left(\sup _{t \leq 1 / n}\left|n^{1 / \alpha}\left(X_{t}-\xi_{t}^{x_{0}}\left(\theta_{0}\right)\right)-n^{1 / \alpha} a\left(x_{0}\right) L_{t}\right|>\varepsilon\right) \leq \begin{cases}C(\varepsilon)\left(1+\left|x_{0}\right|^{p}\right) \frac{\log n}{n^{\alpha}} & \text { if } \alpha<1, \\ C(\varepsilon)\left(1+\left|x_{0}\right|^{p}\right) \frac{1}{n^{1-\delta}}, \forall \delta \in(0,1) \quad \text { if } \alpha \geq 1,\end{cases}
$$

where $C(\varepsilon)$ is a positive constant.

Proof. Recalling that $\left(X_{t}, L_{t}\right)_{t \in[0,1 / n]}=\left(X_{t}^{\tau}, L_{t}^{\tau}\right)_{t \in[0,1 / n]}$ on $\Omega_{n}$ and that $\mathbb{P}\left(\Omega_{n}^{c}\right) \leq 1 / n$, it is sufficient to study the convergence in probability of $\sup _{t \leq 1 / n}\left|n^{1 / \alpha}\left(X_{t}^{\tau}-\xi_{t}^{x_{0}}\left(\theta_{0}\right)\right)-n^{1 / \alpha} a\left(x_{0}\right) L_{t}^{\tau}\right|$. Now using the rescaled 
process $\left(L_{t}^{n}\right)$ this is equivalent to study the convergence in probability of $\sup _{t \in[0,1]} \mid n^{1 / \alpha}\left(Y_{t}^{n}-\xi_{t}^{n, x_{0}}\right)-$ $a\left(x_{0}\right) L_{t}^{n} \mid$. We have the inequality for $t \in[0,1]$

$$
\begin{aligned}
\sup _{s \leq t}\left|n^{1 / \alpha}\left(Y_{s}^{n}-\xi_{s}^{n, x_{0}}\right)-a\left(x_{0}\right) L_{s}^{n}\right| \leq & \frac{\left\|b^{\prime}\right\|_{\infty}}{n} \int_{0}^{t} \sup _{u \leq s}\left|n^{1 / \alpha}\left(Y_{u}^{n}-\xi_{u}^{n, x_{0}}\right)-a\left(x_{0}\right) L_{u}^{n}\right| d s \\
& +\frac{\left\|b^{\prime}\right\|_{\infty}\left|a\left(x_{0}\right)\right|}{n} \sup _{t \in[0,1]}\left|L_{t}^{n}\right|+\sup _{t \in[0,1]}\left|\int_{0}^{t}\left(a\left(Y_{s-}^{n}\right)-a\left(x_{0}\right)\right) d L_{s}^{n}\right|
\end{aligned}
$$

and from Gronwall's inequality we deduce

$$
\sup _{t \in[0,1]}\left|n^{1 / \alpha}\left(Y_{t}^{n}-\xi_{t}^{n, x_{0}}\right)-a\left(x_{0}\right) L_{t}^{n}\right| \leq C\left(\frac{1}{n} \sup _{t \in[0,1]}\left|L_{t}^{n}\right|+\sup _{t \in[0,1]}\left|\int_{0}^{t}\left(a\left(Y_{s-}^{n}\right)-a\left(x_{0}\right)\right) d L_{s}^{n}\right|\right) .
$$

In what follows, we will distinguish between the small jumps and the big jumps of $L_{t}^{n}$. To this end we have

$$
L_{t}^{n}=\int_{0}^{t} \int_{\{0<|z| \leq 1\}} z \tilde{\mu}^{n}(d t, d z)+\int_{0}^{t} \int_{\{|z|>1\}} z \tilde{\mu}^{n}(d t, d z):=L_{t}^{n, 1}+L_{t}^{n, 2}
$$

Control of the small jumps part $L^{n, 1}$

Since $\mathbb{E} \sup _{t \in[0,1]}\left|L_{t}^{n, 1}\right|^{2} \leq C$ we deduce $\mathbb{P}\left(\frac{1}{n} \sup _{t \in[0,1]}\left|L_{t}^{n, 1}\right|>\varepsilon\right) \leq C /\left(n^{2} \varepsilon^{2}\right)$. Turning to the other term $\sup _{t \in[0,1]}\left|\int_{0}^{t}\left(a\left(Y_{s-}^{n}\right)-a\left(x_{0}\right)\right) d L_{s}^{n, 1}\right|$, we have using Lemma 4.1

$$
\mathbb{E}\left(\sup _{t \in[0,1]}\left|\int_{0}^{t}\left(a\left(Y_{s-}^{n}\right)-a\left(x_{0}\right)\right) d L_{s}^{n, 1}\right|^{2}\right) \leq C \mathbb{E}\left(\sup _{t \in[0,1]}\left|Y_{t}^{n}-x_{0}\right|^{2}\right) \leq C\left(1+\left|x_{0}\right|^{2}\right) / n
$$

and we get $\mathbb{P}\left(\sup _{t \in[0,1]}\left|\int_{0}^{t}\left(a\left(Y_{s-}^{n}\right)-a\left(x_{0}\right)\right) d L_{s}^{n, 1}\right|>\varepsilon\right) \leq C(\varepsilon)\left(1+\left|x_{0}\right|^{2}\right) / n$.

Control of the big jumps part $L^{n, 2}$

We distinguish between the cases $\alpha \geq 1$ and $\alpha<1$.

- $\alpha \geq 1$.

Using inequality 2.1.36 in [13] with $\alpha<p<2$, the boundedness of $g$ and the definition of $\tau$, we obtain :

$$
\mathbb{E}\left(\sup _{t \in[0,1]}\left|L_{t}^{n, 2}\right|^{p}\right) \leq C \int_{\left\{1<|z| \leq K(a) n^{1 / \alpha}\right\}} \frac{|z|^{p}}{|z|^{\alpha+1}} d z \leq C n^{p / \alpha} / n
$$

and then from Markov inequality

$$
\mathbb{P}\left(\frac{1}{n} \sup _{t \in[0,1]}\left|L_{t}^{n, 2}\right|>\varepsilon\right) \leq C(\varepsilon) n^{p / \alpha} / n^{p+1} \leq C(\varepsilon) / n .
$$

Similarly we get using Lemma 4.1

$$
\mathbb{E} \sup _{t \in[0,1]}\left|\int_{0}^{t}\left(a\left(Y_{s-}^{n}\right)-a\left(x_{0}\right)\right) d L_{s}^{2, n}\right|^{p} \leq C \mathbb{E}\left(\sup _{t \in[0,1]}\left|Y_{t}^{n}-x_{0}\right|^{p}\right) n^{p / \alpha} / n \leq C\left(1+\left|x_{0}\right|^{p}\right) \frac{n^{p / \alpha}}{n^{2}}
$$

this gives choosing $p=\alpha(1+\delta)$

$$
\mathbb{P}\left(\sup _{t \in[0,1]}\left|\int_{0}^{t}\left(a\left(Y_{s-}^{n}\right)-a\left(x_{0}\right)\right) d L_{s}^{2, n}\right|>\varepsilon\right) \leq C(\varepsilon)\left(1+\left|x_{0}\right|^{p}\right) / n^{1-\delta}
$$

- $\alpha<1$.

Thanks to the symmetry of the compensator $\bar{\mu}^{n}$, we have $L_{t}^{n, 2}=\int_{0}^{t} \int_{|z|>1} z \mu^{n}(d s, d z)$. Then we can write

$$
\left|L_{t}^{n, 2}\right|^{\alpha}=\left|\int_{0}^{t} \int_{\{|z|>1\}} z \mu^{n}(d t, d z)\right|^{\alpha} \leq \int_{0}^{1} \int_{\{|z|>1\}}|z|^{\alpha} \mu^{n}(d t, d z)
$$


consequently $\mathbb{E}\left(\sup _{t \in[0,1]}\left|L_{t}^{n, 2}\right|^{\alpha}\right) \leq C \log n$, and then

$$
\mathbb{P}\left(\frac{1}{n} \sup _{t \in[0,1]}\left|L_{t}^{n, 2}\right|>\varepsilon\right) \leq C(\varepsilon) \frac{\log n}{n^{\alpha}} .
$$

Similarly, we have

$$
\left|\int_{0}^{t}\left(a\left(Y_{s-}^{n}\right)-a\left(x_{0}\right)\right) d L_{s}^{n, 2}\right|^{\alpha} \leq \int_{0}^{1} \int_{\{|z|>1\}}\left|a\left(Y_{s-}^{n}\right)-a\left(x_{0}\right)\right|^{\alpha}|z|^{\alpha} \mu^{n}(d s, d z) .
$$

Taking the expectation and using the Lipschitz assumption on $a$, this yields

$$
\mathbb{E}\left(\sup _{t \in[0,1]}\left|\int_{0}^{t}\left(a\left(Y_{s-}^{n}\right)-a\left(x_{0}\right)\right) d L_{s}^{n, 2}\right|^{\alpha}\right) \leq C \mathbb{E}\left(\sup _{t \in[0,1]}\left|Y_{t}^{n}-x_{0}\right|^{\alpha}\right) \log n \leq C\left(\mathbb{E} \sup _{t \in[0,1]}\left|Y_{t}^{n}-x_{0}\right|\right)^{\alpha} \log n,
$$

where for the second inequality we used Hölder's inequality with $p^{\prime}=1 / \alpha>1$. From Lemma 4.1, we deduce

$$
\mathbb{E}\left(\sup _{t \in[0,1]}\left|\int_{0}^{t}\left(a\left(Y_{s-}^{n}\right)-a\left(x_{0}\right)\right) d L_{s}^{n, 2}\right|^{p}\right) \leq C\left(1+\left|x_{0}\right|^{p}\right) \log n / n^{\alpha} .
$$

and we obtain $\mathbb{P}\left(\sup _{t \in[0,1]}\left|\int_{0}^{t}\left(a\left(Y_{s-}^{n}\right)-a\left(x_{0}\right)\right) d L_{s}^{n}\right|>\varepsilon\right) \leq C(\varepsilon)\left(1+\left|x_{0}\right|^{p}\right) \log n / n^{\alpha}$.

Putting all these results together, Lemma 4.2 is proved.

\subsection{Malliavin Calculus}

In this section, we recall some results on Malliavin calculus for jump processes. We refer to [6] for a complete presentation and to [7] for the adaptation to our framework. We will work on the Poisson space associated to the measure $\mu^{n}$ defining the process $\left(L_{t}^{n}\right)$ of Section 4.1, assuming that $n$ is fixed. By construction, the support of $\mu^{n}$ is contained in $[0,1] \times E_{n}$, where

$$
E_{n}=\left\{z \in \mathbb{R} ;|z|<K(a) n^{1 / \alpha}\right\},
$$

and $K(a)$ is defined by (4.1). We recall that the measure $\mu^{n}$ has compensator

$$
\bar{\mu}^{n}(d t, d z)=d t \frac{g\left(z / n^{1 / \alpha}\right)}{|z|^{\alpha+1}} \tau\left(z / n^{1 / \alpha}\right) 1_{\mathbb{R} \backslash\{0\}} d z:=d t F_{n}(z) d z .
$$

In this section we assume that the truncation function $\tau$ satisfies the additional assumption

$$
\int_{\mathbb{R}}\left|\frac{\tau^{\prime}(z)}{\tau(z)}\right|^{p} \tau(z) d z<\infty, \quad \forall p \geq 1
$$

We define the Malliavin operators $L$ and $\Gamma$ (we omit the dependence in $n$ ) and their basic properties (see Bichteler, Gravereaux, Jacod, [6] Chapter IV, sections 8-9-10). For a test function $f:[0,1] \times \mathbb{R} \mapsto \mathbb{R}(f$ is measurable, $\mathcal{C}^{2}$ with respect to the second variable, with bounded derivatives, and $f \in \cap_{p \geq 1} \mathbf{L}^{p}\left(F_{n}(z) d z\right)$ ), we set $\mu^{n}(f)=\int_{0}^{1} \int_{\mathbb{R}} f(t, z) \mu^{n}(d t, d z)$ (in the sequel this notation is also used for $f: \mathbb{R} \mapsto \mathbb{R}$ ). As auxiliary function, we consider $\rho: \mathbb{R} \mapsto[0, \infty)$ such that $\rho$ is symmetric, two times differentiable and such that $\rho(z)=z^{4}$ if $z \in[0,1 / 2]$ and $\rho(z)=z^{2}$ if $z \geq 1$. Note that thanks to the truncation $\tau$, we do not need that $\rho$ vanishes at infinity. Assuming $\mathrm{H} 2(\mathrm{~b})$, we check that $\rho, \rho^{\prime}$ and $\rho \frac{F_{n}^{\prime}}{F_{n}}$ belong to $\cap_{p \geq 1} \mathbf{L}^{p}\left(F_{n}(z) d z\right)$. With these notations, we define the Malliavin operator $L$, on a simple functional $\mu^{n}(f)$ as follows

$$
L\left(\mu^{n}(f)\right)=\frac{1}{2} \mu^{n}\left(\rho^{\prime} f^{\prime}+\rho \frac{F_{n}^{\prime}}{F_{n}} f^{\prime}+\rho f^{\prime \prime}\right)
$$


where $f^{\prime}$ and $f^{\prime \prime}$ are the derivatives with respect to the second variable. This definition permits to construct a linear operator on a space $D \subset \cap_{p \geq 1} \mathbf{L}^{p}$ which is self-adjoint :

$$
\forall \Phi, \Psi \in D, \quad \mathbb{E} \Phi L \Psi=\mathbb{E} L \Phi \Psi .
$$

We associate to $L$, the symmetric bilinear operator $\Gamma$ :

$$
\Gamma(\Phi, \Psi)=L(\Phi \Psi)-\Phi L \Psi-\Psi L \Phi .
$$

If $f$ and $h$ are two test functions, we have :

$$
\Gamma\left(\mu^{n}(f), \mu^{n}(h)\right)=\mu^{n}\left(\rho f^{\prime} h^{\prime}\right),
$$

The operators $L$ and $\Gamma$ satisfy the chain rule property :

$$
\begin{aligned}
& L F(\Phi)=F^{\prime}(\Phi) L \Phi+\frac{1}{2} F^{\prime \prime}(\Phi) \Gamma(\Phi, \Phi), \\
& \Gamma(F(\Phi), \Psi)=F^{\prime}(\Phi) \Gamma(\Phi, \Psi) .
\end{aligned}
$$

These operators permit to establish the following integration by parts formula (see [6] Theorem 8-10 p.103).

Theorem 4.3. Let $\Phi$ and $\Psi$ be random variables in $D$, and $f$ be a bounded function with bounded derivatives up to order two. If $\Gamma(\Phi, \Phi)$ is invertible and $\Gamma^{-1}(\Phi, \Phi) \in \cap_{p \geq 1} \mathbf{L}^{p}$, we have

$$
\mathbb{E} f^{\prime}(\Phi) \Psi=\mathbb{E} f(\Phi) \mathcal{H}_{\Phi}(\Psi),
$$

with

$$
\mathcal{H}_{\Phi}(\Psi)=-2 \Psi \Gamma^{-1}(\Phi, \Phi) L \Phi-\Gamma\left(\Phi, \Psi \Gamma^{-1}(\Phi, \Phi)\right) .
$$

In the next section, we will apply this Malliavin calculus to the random variables $L_{1}^{n}$ and $Y_{1}^{n}$, which belong to the domain of the operators $L$ and $\Gamma$. From the preceding definitions, we compute easily $L\left(L_{1}^{n}\right)$, $\Gamma\left(L_{1}^{n}, L_{1}^{n}\right)$ and $\mathcal{H}_{L_{1}^{n}}(1)$. We have

$$
\begin{gathered}
L\left(L_{1}^{n}\right)=\frac{1}{2} \mu^{n}\left(\rho^{\prime}+\rho \frac{F_{n}^{\prime}}{F_{n}}\right), \\
\Gamma\left(L_{1}^{n}, L_{1}^{n}\right)=\mu^{n}(\rho), \\
\mathcal{H}_{L_{1}^{n}}(1)=\frac{\Gamma\left(L_{1}^{n}, \Gamma\left(L_{1}^{n}, L_{1}^{n}\right)\right)}{\Gamma\left(L_{1}^{n}, L_{1}^{n}\right) 2}-2 \frac{L\left(L_{1}^{n}\right)}{\Gamma\left(L_{1}^{n}, L_{1}^{n}\right)}=\frac{\mu^{n}\left(\rho \rho^{\prime}\right)}{\mu^{n}(\rho)^{2}}-\frac{\mu^{n}\left(\rho^{\prime}+\rho \frac{F_{n}^{\prime}}{F_{n}}\right)}{\mu^{n}(\rho)} .
\end{gathered}
$$

This leads to the expression

$$
\mathcal{H}_{L_{1}^{n}}(1)=\frac{\mu^{n}\left(\rho \rho^{\prime}\right)}{\mu^{n}(\rho)^{2}}-\frac{\mu^{n}\left(\rho^{\prime}-(\alpha+1) \frac{1}{z} \rho\right)}{\mu^{n}(\rho)}+\frac{1}{n^{1 / \alpha}} R_{n},
$$

where $R_{n}$ is given by

$$
R_{n}=\frac{\mu^{n}\left(\rho \frac{g_{n}^{1}}{g_{n}}\right)}{\mu^{n}(\rho)}+\frac{\mu^{n}\left(\rho \frac{\tau_{n}^{1}}{\tau_{n}}\right)}{\mu^{n}(\rho)}:=R_{n}^{1}+R_{n}^{2},
$$

with the additional notations $g_{n}(z)=g\left(z / n^{1 / \alpha}\right), g_{n}^{1}(z)=g^{\prime}\left(z / n^{1 / \alpha}\right), \tau_{n}(z)=\tau\left(z / n^{1 / \alpha}\right), \tau_{n}^{1}(z)=\tau^{\prime}\left(z / n^{1 / \alpha}\right)$. 
From the choice of $\rho$ we can prove that

$$
\mathbb{E} \frac{1}{\mu^{n}(\rho)^{p}} \leq C_{p}, \quad \forall p \geq 1 .
$$

This is obtained remarking that (see [7] p.2324)

$$
\mathbb{E} \frac{1}{\mu^{n}(\rho)^{p}} \leq \mathbb{E} \frac{1}{\mu^{n}\left(\rho 1_{\{|z| \leq 1 / 2\}}\right)^{p}}=C_{p} \int_{0}^{\infty} u^{p-1} \mathbb{E}\left(e^{-u \mu^{n}\left(\rho 1_{\{|z| \leq 1 / 2\}}\right)}\right) d u .
$$

From the classical exponential formula for Poisson measures, we have

$$
\mathbb{E} e^{-u \mu^{n}\left(\rho 1_{\{|z| \leq 1 / 2\}}\right)}=e^{-\int_{\{|z| \leq 1 / 2\}}\left(1-e^{-u \rho(z)}\right) \frac{g_{n}(z)}{|z|^{\alpha+1}} \tau_{n}(z) d z} \leq e^{-\int_{\{|z| \leq 1 / 2\}}\left(1-e^{-u z^{4}}\right) \frac{C}{|z|^{\alpha+1}} d z},
$$

where we used that $g$ is lower bounded by $C>0$ in a neighborhood of zero (recall that $g$ is continuous and $g(0)=1), \tau=1$ and $\rho(z)=z^{4}$ on $\{|z| \leq 1 / 2\}$. We conclude observing that

$\liminf _{u \rightarrow \infty} \frac{1}{\ln u} \int_{\{|z| \leq 1 / 2\}} 1_{\left\{z^{4} \geq 1 / u\right\}} \frac{C}{|z|^{\alpha+1}} d z=+\infty$.

Moreover on $E_{n}$ we observe that $|z| / n^{1 / \alpha} \leq \eta$ and then assuming $\mathrm{H} 2(\mathrm{~b})$ we have the bound

$$
\left|R_{n}^{1}\right| \leq C
$$

Turning to $R_{n}^{2}$, and using the definition of $\tau$ we have

$$
\left|R_{n}^{2}\right| \leq \frac{\mu_{n}\left(\rho\left|\frac{\tau_{n}^{1}}{\tau_{n}}\right| 1_{\left\{K(a) n^{1 / \alpha} / 2<|z|<K(a) n^{1 / \alpha}\right\}}\right)}{\mu_{n}\left(\rho 1_{\left\{K(a) n^{1 / \alpha} / 2<|z|<K(a) n^{1 / \alpha}\right\}}\right)} \leq \mu_{n}\left(\left|\frac{\tau_{n}^{1}}{\tau_{n}}\right| 1_{\left\{K(a) n^{1 / \alpha} / 2<|z|<K(a) n^{1 / \alpha}\right\}}\right) .
$$

Since $\mu_{n}=\tilde{\mu}_{n}+\bar{\mu}_{n}$, we deduce from inequalities 2.1.36 and 2.1.37 in [13], the change of variable $u=z / n^{1 / \alpha}$ and assumption (4.15) that

$$
\mathbb{E}\left|R_{n}^{2}\right|^{p} \leq C_{p} / n, \quad \forall p \geq 1 .
$$

This permits to deduce the following useful inequalities.

Lemma 4.3. We have

$$
\begin{aligned}
& \sup _{n} \mathbb{E}\left|\mathcal{H}_{L_{1}^{n}}(1)\right|^{p} \leq C_{p}, \quad \forall p \geq 1, \\
& \sup _{n} \mathbb{E}\left|\int_{0}^{1} \int_{\{|z|>1\}}\right| z\left|\mu^{n}(d s, d z) \mathcal{H}_{L_{1}^{n}}(1)\right|^{p} \leq C_{p}, \quad \forall p \geq 1, \\
& \sup _{n} \mathbb{E} \sup _{t \in[0,1]}\left|L_{t}^{n}\right| \mid \mathcal{H}_{L_{1}^{n}}(1) \leq C .
\end{aligned}
$$

Proof. Obviously (4.30) is a consequence of (4.28) and (4.29). From (4.26) and (4.27), to prove (4.28) we just have to consider the first two terms in the right-hand side of (4.24). Distinguishing between the small jumps and the big jumps of the Poisson measure we have for the first term

$$
\frac{\mu^{n}\left(\rho \rho^{\prime}\right)}{\mu^{n}(\rho)^{2}} \leq \frac{\mu^{n}\left(\rho \rho^{\prime} 1_{\{|z|<1\}}\right)}{\mu^{n}(\rho)^{2}}+\frac{\mu^{n}\left(\rho \rho^{\prime} 1_{\{|z| \geq 1\}}\right)}{\mu^{n}\left(\rho 1_{\{|z| \geq 1\}}\right)^{2}} .
$$

We conclude immediately using (4.25) that $\forall p \geq 1, \sup _{n} \mathbb{E}\left|\frac{\mu^{n}\left(\rho \rho^{\prime} 1_{\{|z|<1\}}\right)}{\mu^{n}(\rho)^{2}}\right|^{p} \leq C_{p}$. Moreover, recalling that $\rho(z)=z^{2}$ for $|z| \geq 1$ we deduce that $\frac{\mu^{n}\left(\rho \rho^{\prime} 1_{\{|z| \geq 1\}}\right)}{\mu^{n}\left(\rho 1_{\{|z| \geq 1\}}\right)^{2}} \leq 2$ and this yields $\forall p \geq 1, \sup _{n} \mathbb{E}\left|\frac{\mu^{n}\left(\rho \rho^{\prime}\right)}{\mu^{n}(\rho)^{2}}\right|^{p} \leq C_{p}$. We proceed similarly for $\frac{\mu^{n}\left(\rho^{\prime}-(\alpha+1) \frac{1}{z} \rho\right)}{\mu^{n}(\rho)}$ and this achieves the proof of (4.28). 
It remains to prove (4.29). We check immediately from (4.26) and (4.27) that

$$
\mathbb{E}\left|\int_{0}^{1} \int_{\{|z|>1\}}\right| z\left|\mu^{n}(d s, d z) \frac{1}{n^{1 / \alpha}} R_{n}\right|^{p} \leq C_{p} .
$$

Turning to $\int_{0}^{1} \int_{\{|z|>1\}}|z| \mu^{n}(d s, d z) \frac{\mu^{n}\left(\rho \rho^{\prime}\right)}{\mu^{n}(\rho)^{2}}$, from the Cauchy-Schwarz inequality (and using $\rho(z)=z^{2}$ if $|z| \geq 1)$ we get $\int_{0}^{1} \int_{\{|z|>1\}}|z| \mu^{n}(d s, d z) \leq \mu_{n}\left(1_{\{|z| \geq 1\}}\right)^{1 / 2} \mu_{n}\left(\rho 1_{\{|z| \geq 1\}}\right)^{1 / 2}$ and we deduce the bound

$$
\int_{0}^{1} \int_{\{|z|>1\}}|z| \mu^{n}(d s, d z) \frac{\mu^{n}\left(\left|\rho \rho^{\prime}\right|\right)}{\mu^{n}(\rho)^{2}} \leq \mu_{n}\left(1_{\{|z| \geq 1\}}\right)^{1 / 2} \frac{\mu^{n}\left(\left|\rho \rho^{\prime}\right|\right)}{\mu^{n}(\rho)^{3 / 2}} .
$$

Remarking that $\mu_{n}\left(1_{\{|z| \geq 1\}}\right)$ has a Poisson distribution with some parameter $\lambda_{\alpha}^{n}$ bounded by $\lambda_{\alpha}$ independent of $n$ we get that

$$
\sup _{n} \mathbb{E}\left|\mu_{n}\left(1_{\{|z| \geq 1\}}\right)^{1 / 2} \frac{\mu^{n}\left(\left|\rho \rho^{\prime}\right| 1_{\{|z|<1\}}\right)}{\mu^{n}(\rho)^{3 / 2}}\right|^{p} \leq C_{p}, \quad \forall p \geq 1 .
$$

Considering the large jumps part, we have

$$
\mu_{n}\left(1_{\{|z| \geq 1\}}\right)^{1 / 2} \frac{\mu^{n}\left(\left|\rho \rho^{\prime}\right| 1_{\{|z| \geq 1\}}\right)}{\mu^{n}(\rho)^{3 / 2}} \leq \mu_{n}\left(1_{\{|z| \geq 1\}}\right)^{1 / 2} \frac{\mu^{n}\left(\left|\rho \rho^{\prime}\right| 1_{\{|z| \geq 1\}}\right)}{\mu^{n}\left(\rho 1_{\{|z| \geq 1\}}\right)^{3 / 2}} \leq 2 \mu_{n}\left(1_{\{|z| \geq 1\}}\right)^{1 / 2},
$$

and this permits to conclude that

$$
\sup _{n} \mathbb{E}\left|\int_{0}^{1} \int_{\{|z|>1\}}\right| z\left|\mu^{n}(d s, d z) \frac{\mu^{n}\left(\rho \rho^{\prime}\right)}{\mu^{n}(\rho)^{2}}\right|^{p} \leq C_{p}, \quad \forall p \geq 1 .
$$

In the same way, we have for the last term

$$
\int_{0}^{1} \int_{\{|z|>1\}}|z| \mu^{n}(d s, d z)\left|\frac{\mu^{n}\left(\rho^{\prime}-(\alpha+1) \frac{1}{z} \rho\right)}{\mu^{n}(\rho)}\right| \leq C \mu_{n}\left(1_{\{|z| \geq 1\}}\right)^{1 / 2} \frac{\mu^{n}\left(\left|\rho^{\prime}+\rho / z\right|\right)}{\mu^{n}(\rho)^{1 / 2}} .
$$

We conclude as previously remarking that for the large jumps part we have, using once again the CauchySchwarz inequality,

$$
\mu_{n}\left(1_{\{|z| \geq 1\}}\right)^{1 / 2} \frac{\mu^{n}\left(\left|\rho^{\prime}+\rho / z\right| 1_{\{|z| \geq 1\}}\right)}{\mu^{n}(\rho)^{1 / 2}} \leq 3 \mu_{n}\left(1_{\{|z| \geq 1\}}\right) .
$$

This ends the proof of (4.29).

With this background, we can proceed to the proof of Theorems 4.1 and 4.2 .

\subsection{Proof of Theorem 4.1}

From the the localization and rescaling procedure, we just have to prove

$$
\left|\mathbb{E} h\left(n^{1 / \alpha}\left(Y_{1}^{n}-\xi_{1}^{n, x_{0}}\right)\right)-\mathbb{E} h\left(a\left(x_{0}\right) L_{1}^{n}\right)\right| \leq C\left(1+\left|x_{0}\right|\right) \varepsilon_{n}\|h\|_{\infty} .
$$

Now, considering a regularizing sequence $\left(h_{p}\right)$ converging to $h$ in $L^{1}$-norm, such that $\forall p, h_{p}$ admits a bounded derivative and $\left\|h_{p}\right\|_{\infty} \leq\|h\|_{\infty}$, we may assume that $h$ admits a bounded derivative.

Using the integration by part formula (4.21) and denoting by $H$ any primitive function of $h$

$$
\mathbb{E} h\left(a\left(x_{0}\right) L_{1}^{n}\right)=\mathbb{E} H\left(a\left(x_{0}\right) L_{1}^{n}\right) \mathcal{H}_{a\left(x_{0}\right) L_{1}^{n}}(1),
$$

and then from the triangle inequality, we have to bound the two following terms :

$$
T_{1}:=\left|\mathbb{E} h\left(n^{1 / \alpha}\left(Y_{1}^{n}-\xi_{1}^{n, x_{0}}\right)\right)-\mathbb{E} H\left(n^{1 / \alpha}\left(Y_{1}^{n}-\xi_{1}^{n, x_{0}}\right)\right) \mathcal{H}_{a\left(x_{0}\right) L_{1}^{n}}(1)\right|
$$




$$
T_{2}:=\left|\mathbb{E} H\left(n^{1 / \alpha}\left(Y_{1}^{n}-\xi_{1}^{n, x_{0}}\right)\right) \mathcal{H}_{a\left(x_{0}\right) L_{1}^{n}}(1)-\mathbb{E} H\left(a\left(x_{0}\right) L_{1}^{n}\right) \mathcal{H}_{a\left(x_{0}\right) L_{1}^{n}}(1)\right| .
$$

Bound for $T_{2}$

We have

$$
T_{2} \leq\|h\|_{\infty} \mathbb{E}\left|n^{1 / \alpha}\left(Y_{1}^{n}-\xi_{1}^{n, x_{0}}\right)-a\left(x_{0}\right) L_{1}^{n}\right|\left|\mathcal{H}_{a\left(x_{0}\right) L_{1}^{n}}(1)\right|,
$$

and from (4.11) we get

$$
T_{2} \leq\|h\|_{\infty} \frac{1}{\left|a\left(x_{0}\right)\right|}\left(\frac{1}{n} \mathbb{E} \sup _{t \in[0,1]}\left|L_{t}^{n}\right|\left|\mathcal{H}_{L_{1}^{n}}(1)\right|+\mathbb{E} \sup _{t \in[0,1]}\left|\int_{0}^{t}\left(a\left(Y_{s-}^{n}\right)-a\left(x_{0}\right)\right) d L_{s}^{n}\right|\left|\mathcal{H}_{L_{1}^{n}}(1)\right|\right),
$$

where as a consequence of the linearity property of the operators $\Gamma$ and $L$, we have used $\mathcal{H}_{a\left(x_{0}\right) L_{1}^{n}(1)}=$ $\frac{1}{a\left(x_{0}\right)} \mathcal{H}_{L_{1}^{n}}(1)$.

From (4.30), the first term in the right-hand side of the above inequality is bounded by $C / n$.

Turning to the second term $\mathbb{E} \sup _{t \in[0,1]}\left|\int_{0}^{t}\left(a\left(Y_{s-}^{n}\right)-a\left(x_{0}\right)\right) d L_{s}^{n}\right|\left|\mathcal{H}_{L_{1}^{n}}(1)\right|$, we use once again the decomposition (4.12). For the small jumps part, Hölder's inequality and inequality 2.1 .36 in [13] with $1<p<2$, $p>\alpha$ and $q$ such that $1 / p+1 / q=1$, lead to

$$
\mathbb{E} \sup _{t \in[0,1]}\left|\int_{0}^{t}\left(a\left(Y_{s-}^{n}\right)-a\left(x_{0}\right)\right) d L_{s}^{n, 1}\right|\left|\mathcal{H}_{L_{1}^{n}}(1)\right| \leq C_{p}\left(\mathbb{E} \sup _{t \in[0,1]}\left|a\left(Y_{t-}^{n}\right)-a\left(x_{0}\right)\right|^{p}\right)^{1 / p}\left(\mathbb{E}\left|\mathcal{H}_{L_{1}^{n}}(1)\right|^{q}\right)^{1 / q} .
$$

From the Lipschitz assumption on $a$, the result of Lemma 4.1 and (4.28), we conclude

$$
\mathbb{E} \sup _{t \in[0,1]}\left|\int_{0}^{t}\left(a\left(Y_{s-}^{n}\right)-a\left(x_{0}\right)\right) d L_{s}^{n, 1}\right|\left|\mathcal{H}_{L_{1}^{n}}(1)\right| \leq C_{p}\left(1+\left|x_{0}\right|\right) \frac{1}{n^{1 / p}} .
$$

This gives the bound

$$
\mathbb{E} \sup _{t \in[0,1]}\left|\int_{0}^{t}\left(a\left(Y_{s-}^{n}\right)-a\left(x_{0}\right)\right) d L_{s}^{n, 1}\right|\left|\mathcal{H}_{L_{1}^{n}}(1)\right| \leq C_{p}\left(1+\left|x_{0}\right|\right) \varepsilon_{n},
$$

with $\varepsilon_{n}$ given as in Theorem 4.1 .

For the big jumps part, we remark first that

$$
\int_{0}^{t}\left(a\left(Y_{s-}^{n}\right)-a\left(x_{0}\right)\right) d L_{s}^{n, 2}=\int_{0}^{t} \int_{\{|z|>1\}}\left(a\left(Y_{s-}^{n}\right)-a\left(x_{0}\right)\right) z \mu^{n}(d t, d z)
$$

and then

$$
\mathbb{E} \sup _{t \in[0,1]}\left|\int_{0}^{t}\left(a\left(Y_{s-}^{n}\right)-a\left(x_{0}\right)\right) d L_{s}^{n, 2}\right|\left|\mathcal{H}_{L_{1}^{n}}(1)\right| \leq \mathbb{E}\left[\sup _{t \in[0,1]}\left|a\left(Y_{t-}^{n}\right)-a\left(x_{0}\right)\right| \int_{0}^{t} \int_{\{|z|>1\}}|z| \mu^{n}(d t, d z) \mid \mathcal{H}_{L_{1}^{n}}(1)\right] .
$$

As for the small jumps part, we conclude applying successively Hölder's inequality, Lemma 4.1 and (4.29) and this shows finally

$$
T_{2} \leq C\left(1+\left|x_{0}\right|\right) \varepsilon_{n}
$$

Bound for $T_{1}$

It remains to consider (4.31).

From (4.22) and (4.17), we remark that

$$
\mathcal{H}_{a\left(x_{0}\right) L_{1}^{n}}(1)=L\left(\frac{1}{\Gamma\left(a\left(x_{0}\right) L_{1}^{n}, a\left(x_{0}\right) L_{1}^{n}\right)}\right) a\left(x_{0}\right) L_{1}^{n}-\frac{L\left(a\left(x_{0}\right) L_{1}^{n}\right)}{\Gamma\left(a\left(x_{0}\right) L_{1}^{n}, a\left(x_{0}\right) L_{1}^{n}\right)}-L\left(\frac{a\left(x_{0}\right) L_{1}^{n}}{\Gamma\left(a\left(x_{0}\right) L_{1}^{n}, a\left(x_{0}\right) L_{1}^{n}\right)}\right),
$$


and using the self-adjoint property of the operator $L$ for the first and third terms we obtain

$$
\begin{aligned}
& \mathbb{E} H\left(n^{1 / \alpha}\left(Y_{1}^{n}-\xi_{1}^{n, x_{0}}\right)\right) \mathcal{H}_{a\left(x_{0}\right) L_{1}^{n}}(1)= \\
& \mathbb{E}\left(\frac{L\left(H\left(n^{1 / \alpha}\left(Y_{1}^{n}-\xi_{1}^{n, x_{0}}\right)\right) a\left(x_{0}\right) L_{1}^{n}\right)-H\left(n^{1 / \alpha}\left(Y_{1}^{n}-\xi_{1}^{n, x_{0}}\right)\right) L\left(a\left(x_{0}\right) L_{1}^{n}\right)-L\left(H\left(n^{1 / \alpha}\left(Y_{1}^{n}-\xi_{1}^{n, x_{0}}\right)\right)\right) a\left(x_{0}\right) L_{1}^{n}}{\Gamma\left(a\left(x_{0}\right) L_{1}^{n}, a\left(x_{0}\right) L_{1}^{n}\right)}\right) .
\end{aligned}
$$

Using again (4.17) and the chain rule we get

$$
\mathbb{E} H\left(n^{1 / \alpha}\left(Y_{1}^{n}-\xi_{1}^{n, x_{0}}\right)\right) \mathcal{H}_{a\left(x_{0}\right) L_{1}^{n}}(1)=\mathbb{E} h\left(n^{1 / \alpha}\left(Y_{1}^{n}-\xi_{1}^{n, x_{0}}\right)\right) \frac{\Gamma\left(n^{1 / \alpha}\left(Y_{1}^{n}-\xi_{1}^{n, x_{0}}\right), a\left(x_{0}\right) L_{1}^{n}\right)}{\Gamma\left(a\left(x_{0}\right) L_{1}^{n}, a\left(x_{0}\right) L_{1}^{n}\right)}
$$

and so

$$
T_{1} \leq\|h\|_{\infty} \mathbb{E}\left|\frac{\Gamma\left(n^{1 / \alpha}\left(Y_{1}^{n}-\xi_{1}^{n, x_{0}}\right), a\left(x_{0}\right) L_{1}^{n}\right)}{\Gamma\left(a\left(x_{0}\right) L_{1}^{n}, a\left(x_{0}\right) L_{1}^{n}\right)}-1\right| .
$$

From the linearity property of $\Gamma$ and since $\xi_{1}^{n, x_{0}}$ is deterministic, we have $\Gamma\left(n^{1 / \alpha}\left(Y_{1}^{n}-\xi_{1}^{n, x_{0}}\right), a\left(x_{0}\right) L_{1}^{n}\right)=$ $n^{1 / \alpha} a\left(x_{0}\right) \Gamma\left(Y_{1}^{n}, L_{1}^{n}\right)$ and $\Gamma\left(a\left(x_{0}\right) L_{1}^{n}, a\left(x_{0}\right) L_{1}^{n}\right)=a\left(x_{0}\right)^{2} \Gamma\left(L_{1}^{n}, L_{1}^{n}\right)$.

Setting $U_{t}^{n}=n^{1 / \alpha} \Gamma\left(Y_{t}^{n}, L_{t}^{n}\right), t \in[0,1]$, this leads to the simplification

$$
\left|\frac{\Gamma\left(n^{1 / \alpha}\left(Y_{1}^{n}-\xi_{1}^{n, x_{0}}\right), a\left(x_{0}\right) L_{1}^{n}\right)}{\Gamma\left(a\left(x_{0}\right) L_{1}^{n}, a\left(x_{0}\right) L_{1}^{n}\right)}-1\right|=\frac{1}{\left|a\left(x_{0}\right)\right|} \frac{\left|U_{1}^{n}-a\left(x_{0}\right) \int_{0}^{1} \int_{E_{n}} \rho(z) \mu^{n}(d s, d z)\right|}{\int_{0}^{1} \int_{E_{n}} \rho(z) \mu^{n}(d s, d z)},
$$

and

$$
T_{1} \leq\|h\|_{\infty} \frac{1}{\left|a\left(x_{0}\right)\right|} \mathbb{E}\left(\frac{\left|U_{1}^{n}-a\left(x_{0}\right) \int_{0}^{1} \int_{E_{n}} \rho(z) \mu^{n}(d s, d z)\right|}{\int_{0}^{1} \int_{E_{n}} \rho(z) \mu^{n}(d s, d z)}\right) .
$$

From Theorem 10-3 p.130 in [6], we can prove that the process $\left(U_{t}^{n}\right)$ solves

$$
U_{t}^{n}=\frac{1}{n} \int_{0}^{t} b^{\prime}\left(Y_{s}^{n}\right) U_{s}^{n} d s+\frac{1}{n^{1 / \alpha}} \int_{0}^{t} \int_{E_{n}} a^{\prime}\left(Y_{s-}^{n}\right) U_{s-}^{n} z \tilde{\mu}^{n}(d s, d z)+\int_{0}^{t} \int_{E_{n}} a\left(Y_{s-}^{n}\right) \rho(z) \mu^{n}(d s, d z), \quad t \in[0,1] .
$$

Introducing the linear stochastic equation

$$
Z_{t}^{n}=1+\frac{1}{n} \int_{0}^{t} b^{\prime}\left(Y_{s}^{n}\right) Z_{s}^{n} d s+\frac{1}{n^{1 / \alpha}} \int_{0}^{t} \int_{E_{n}} a^{\prime}\left(Y_{s-}^{n}\right) Z_{s-}^{n} z \tilde{\mu}^{n}(d s, d z), \quad t \in[0,1],
$$

and using Itô's formula, we show that $Z_{t}^{n}$ admits an inverse, denoted by $\left(Z_{t}^{n}\right)^{-1}$, that solves

$$
\begin{aligned}
\left(Z_{t}^{n}\right)^{-1}=1-\frac{1}{n} \int_{0}^{t} b^{\prime}\left(Y_{s}^{n}\right)\left(Z_{s}^{n}\right)^{-1} d s & -\frac{1}{n^{1 / \alpha}} \int_{0}^{t} \int_{E_{n}} \frac{a^{\prime}\left(Y_{s-}^{n}\right) z}{1+a^{\prime}\left(Y_{s-}^{n}\right) z / n^{1 / \alpha}}\left(Z_{s-}^{n}\right)^{-1} \tilde{\mu}^{n}(d s, d z) \\
& +\frac{1}{n^{2 / \alpha}} \int_{0}^{t} \int_{E_{n}} \frac{\left(a^{\prime}\left(Y_{s-}^{n}\right) z\right)^{2}}{1+a^{\prime}\left(Y_{s-}^{n}\right) z / n^{1 / \alpha}}\left(Z_{s-}^{n}\right)^{-1} \bar{\mu}^{n}(d s, d z), \quad t \in[0,1] .
\end{aligned}
$$

Note that on $E_{n}, 0<\frac{1}{1+a^{\prime}\left(Y_{s-}^{n}\right) z / n^{1 / \alpha}} \leq 2$ and the above integrals are well defined.

With these processes we can solve (4.34) and we obtain the explicit expression

$$
U_{t}^{n}=Z_{t}^{n} \int_{0}^{t} \int_{E_{n}} \frac{a\left(Y_{s-}^{n}\right) \rho(z)}{1+a^{\prime}\left(Y_{s-}^{n}\right) z / n^{1 / \alpha}}\left(Z_{s-}^{n}\right)^{-1} \mu^{n}(d s, d z), \quad t \in[0,1] .
$$

Moreover we can prove the following bounds for the processes $\left(Z^{n}\right)$ and $\left(Z^{n}\right)^{-1}$. 
Lemma 4.4. Let $p \geq 1$ and $p>\alpha$, then

$$
\begin{aligned}
& \mathbb{E} \sup _{t \in[0,1]}\left|Z_{t}^{n}-1\right|^{p} \leq C_{p} / n, \\
& \mathbb{E} \sup _{t \in[0,1]}\left|\left(Z_{t}^{n}\right)^{-1}-1\right|^{p} \leq C_{p} / n .
\end{aligned}
$$

The result of Lemma 4.4 follows from convexity inequality, inequality 2.1.36 (or 2.1.37 if $p \geq 2$ ) in [13] and Gronwall's Lemma. We omit its standard proof.

Plugging (4.37) into (4.33), we split the right-hand side of (4.33) into four parts :

$$
\begin{aligned}
& T_{1,1}=\mathbb{E}\left(\frac{\left|Z_{1}^{n} \int_{0}^{1} \int_{E_{n}} \frac{\left(a\left(Y_{s-}^{n}\right)-a\left(x_{0}\right)\right) \rho(z)}{1+a^{\prime}\left(Y_{s-}^{n}\right) z / n^{1 / \alpha}}\left(Z_{s-}^{n}\right)^{-1} \mu^{n}(d s, d z)\right|}{\int_{0}^{1} \int_{E_{n}} \rho(z) \mu^{n}(d s, d z)}\right), \\
& T_{1,2}=\mathbb{E}\left(\frac{\left|Z_{1}^{n}-1\right|\left|\int_{0}^{1} \int_{E_{n}} \frac{a\left(x_{0}\right) \rho(z)}{1+a^{\prime}\left(Y_{s-}^{n}\right) z / n^{1 / \alpha}}\left(Z_{s-}^{n}\right)^{-1} \mu^{n}(d s, d z)\right|}{\int_{0}^{1} \int_{E_{n}} \rho(z) \mu^{n}(d s, d z)}\right), \\
& T_{1,3}=\mathbb{E}\left(\frac{\left|\int_{0}^{1} \int_{E_{n}} \frac{\left(\left(Z_{s-}^{n}\right)^{-1}-1\right)}{1+a^{\prime}\left(Y_{s-}^{n}\right) z / n^{1 / \alpha}} a\left(x_{0}\right) \rho(z) \mu^{n}(d s, d z)\right|}{\int_{0}^{1} \int_{E_{n}} \rho(z) \mu^{n}(d s, d z)}\right), \\
& T_{1,4}=\mathbb{E}\left(\frac{\left|\int_{0}^{1} \int_{E_{n}} \frac{a^{\prime}\left(Y_{s-}^{n}\right) z / n^{1 / \alpha}}{1+a^{\prime}\left(Y_{s-}^{n}\right) z / n^{1 / \alpha}} a\left(x_{0}\right) \rho(z) \mu^{n}(d s, d z)\right|}{\int_{0}^{1} \int_{E_{n}} \rho(z) \mu^{n}(d s, d z)}\right) .
\end{aligned}
$$

Considering first $T_{1,1}$, we have

$$
T_{1,1} \leq 2 \mathbb{E}\left(\left|Z_{1}^{n}\right| \sup _{t \in[0,1]}\left|\left(Z_{t}^{n}\right)^{-1}\right| \sup _{t \in[0,1]}\left|a\left(Y_{t-}^{n}\right)-a\left(x_{0}\right)\right|\right),
$$

and from Hölder's inequality with conjugated $p$ and $q$ such that $1<p<2$ and $p>\alpha$ we obtain from Lemma 4.1 (with $p$ ) and Lemma 4.4 (with $q$ )

$$
T_{1,1} \leq C_{p}\left(1+\left|x_{0}\right|\right) \varepsilon_{n},
$$

where $\varepsilon_{n}$ is defined in Theorem 4.1. Turning to $T_{1,2}$, we have

$$
T_{1,2} \leq 2\left|a\left(x_{0}\right)\right| \mathbb{E}\left(\left|Z_{1}^{n}-1\right| \sup _{t \in[0,1]}\left|\left(Z_{t}^{n}\right)^{-1}\right|\right),
$$

and as previously using Lemma 4.4, we deduce

$$
T_{1,2} \leq C_{p}\left(1+\left|x_{0}\right|\right) \varepsilon_{n}
$$

The third term satisfies $T_{1,3} \leq 2\left|a\left(x_{0}\right)\right| \mathbb{E}\left(\sup _{t \in[0,1]}\left|\left(Z_{t}^{n}\right)^{-1}-1\right|\right)$ and so

$$
T_{1,3} \leq C_{p}\left(1+\left|x_{0}\right|\right) \varepsilon_{n}
$$

Finally for the last term, we observe that

$$
\begin{aligned}
T_{1,4} & \leq 2\left|a\left(x_{0}\right)\right|\left\|a^{\prime}\right\|_{\infty} \mathbb{E}\left(\frac{\int_{0}^{1} \int_{E_{n}}\left(|z| / n^{1 / \alpha}\right) \rho(z) \mu^{n}(d s, d z)}{\int_{0}^{1} \int_{E_{n}} \rho(z) \mu^{n}(d s, d z)}\right) \\
& \leq C\left(1+\left|x_{0}\right|\right)\left(\frac{1}{n^{1 / \alpha}}+\mathbb{E}\left(\frac{\int_{0}^{1} \int_{E_{n} \cap\{|z|>1\}}\left(|z| / n^{1 / \alpha}\right) \rho(z) \mu^{n}(d s, d z)}{\int_{0}^{1} \int_{E_{n} \cap\{|z|>1\}} \rho(z) \mu^{n}(d s, d z)}\right)\right) .
\end{aligned}
$$


But remarking that

$$
\frac{\int_{0}^{1} \int_{E_{n} \cap\{|z|>1\}}\left(|z| / n^{1 / \alpha}\right) \rho(z) \mu^{n}(d s, d z)}{\int_{0}^{1} \int_{E_{n} \cap\{|z|>1\}} \rho(z) \mu^{n}(d s, d z)} \leq \int_{0}^{1} \int_{E_{n} \cap\{|z|>1\}}\left(|z| / n^{1 / \alpha}\right) \mu^{n}(d s, d z)
$$

and taking the expectation we deduce that if $\alpha \neq 1$

$$
\mathbb{E} \frac{\int_{0}^{1} \int_{E_{n} \cap\{|z|>1\}}\left(|z| / n^{1 / \alpha}\right) \rho(z) \mu^{n}(d s, d z)}{\int_{0}^{1} \int_{E_{n} \cap\{|z|>1\}} \rho(z) \mu^{n}(d s, d z)} \leq C / n,
$$

and if $\alpha=1$

$$
\mathbb{E} \frac{\int_{0}^{1} \int_{E_{n} \cap\{|z|>1\}}\left(|z| / n^{1 / \alpha}\right) \rho(z) \mu^{n}(d s, d z)}{\int_{0}^{1} \int_{E_{n} \cap\{|z|>1\}} \rho(z) \mu^{n}(d s, d z)} \leq C \log n / n
$$

This yields to

$$
T_{1,4} \leq C \varepsilon_{n}
$$

Combining all these results we obtain

$$
T_{1} \leq C\left(1+\left|x_{0}\right|\right) \varepsilon_{n}
$$

and the proof of Theorem 4.1 is finished.

\subsection{Proof of Theorem 4.2}

As in the proof of Theorem 4.1 we will use Malliavin calculus integration by part formula. The first step is to construct on the same probability space two random variables whose laws are close to the laws of $n^{1 / \alpha} L_{1 / n}$ and $L_{1}^{\alpha}$. We recall briefly the notations of Section $4.1: \mu^{n}$ is a Poisson random measure with compensator $\bar{\mu}^{n}(d t, d z)=d t \frac{g\left(z / n^{1 / \alpha}\right)}{|z|^{\alpha+1}} \tau\left(z / n^{1 / \alpha}\right) 1_{\mathbb{R} \backslash\{0\}}(z) d z$, where $\tau$ is a truncation function, and the process $\left(L_{t}^{n}\right)_{t}$ defined by $L_{t}^{n}=\int_{0}^{t} \int_{\mathbb{R}} z \tilde{\mu}^{n}(d s, d z)$, with $\tilde{\mu}^{n}=\mu^{n}-\bar{\mu}^{n}$ is such that (see (4.3), (4.8))

$$
\left|\mathbb{E}\left[h\left(n^{1 / \alpha} L_{1 / n}\right)\right]-\mathbb{E}\left[h\left(L_{1}^{n}\right)\right]\right| \leq C \frac{\|h\|_{\infty}}{n} .
$$

We now construct a variable approximating the law of $L_{1}^{\alpha}$, and based on the Poisson measure $\mu^{n}$.

For $x>0$ we define

$$
G(x)=\int_{x}^{\infty} \frac{g(z) \tau(z)}{z^{1+\alpha}} d z, \text { and, } H(x)=\int_{x}^{\infty} \frac{\tau(z)}{z^{1+\alpha}} d z .
$$

Recall that $\tau$ is a truncation function equal to 1 on $[-K(a) / 2, K(a) / 2]$ and equal to 0 on $[-K(a), K(a)]^{c}$. We assume for the sequel of the proof that $\tau(z)>0, g(z)>0$, for $|z|<K(a)$. Indeed, $\tau$ is a truncation function that can be chosen non vanishing on $(-K(a), K(a))$, and up to reducing the value of $\eta$ in the Assumption H2(b) we can assume that $g$ does not vanish on $[-K(a), K(a)]$. Then, it is immediate to check that $G$ and $H$ are non increasing, one to one, functions from $(0, K(a)]$ to $(\infty, 0]$. We define $s_{n}(z)=$ $n^{1 / \alpha} G^{-1}\left(H\left(n^{-1 / \alpha} z\right)\right)$ for $z \in\left(0, n^{1 / \alpha} K(a)\right], s_{n}(0)=0$, and $s_{n}(z)=-s_{n}(-z)$ for $z \in\left[-n^{1 / \alpha} K(a), 0\right)$. The function $s_{n}$ is increasing, odd and one to one, from the interval $\left[-n^{1 / \alpha} K(a), n^{1 / \alpha} K(a)\right]$ on itself and we let $h_{n}=s_{n}^{-1}:\left[-n^{1 / \alpha} K(a), n^{1 / \alpha} K(a)\right] \rightarrow\left[-n^{1 / \alpha} K(a), n^{1 / \alpha} K(a)\right]$ be its inverse function. Let us admit temporarily the next lemma about the behaviour of the functions $h_{n}$ as $n \rightarrow \infty$.

Lemma 4.5. 1) There exists $\varepsilon>0$ such that, for $|z| \leq \varepsilon n^{1 / \alpha}$,

$$
\begin{aligned}
& \left|h_{n}(z)-z\right| \leq C \frac{z^{2}}{n^{1 / \alpha}}+C \frac{|z|^{\alpha+1}}{n}, \quad \text { if } \alpha \neq 1, \\
& \left|h_{n}(z)-z\right| \leq C \frac{z^{2}}{n}\left|\log \left(\frac{|z|}{n}\right)\right|, \quad \text { if } \alpha=1 .
\end{aligned}
$$


2) The function $h_{n}$ is $\mathcal{C}^{1}$ on $\left(-\varepsilon n^{1 / \alpha}, \varepsilon n^{1 / \alpha}\right)$ and for $|z|<\varepsilon n^{1 / \alpha}$,

$$
\begin{aligned}
& \left|h_{n}^{\prime}(z)-1\right| \leq C \frac{|z|}{n^{1 / \alpha}}+C \frac{|z|^{\alpha}}{n}, \quad \text { if } \alpha \neq 1, \\
& \left|h_{n}^{\prime}(z)-1\right| \leq C \frac{|z|}{n}\left|\log \left(\frac{|z|}{n}\right)\right|, \quad \text { if } \alpha=1 .
\end{aligned}
$$

Using the previous lemma, we can define a process $\left(L_{t}^{\alpha, n}\right)_{t \in[0,1]}$ by setting

$$
L_{t}^{\alpha, n}=\int_{0}^{t} \int_{\left\{|z| \leq n^{1 / \alpha} K(a)\right\}} h_{n}(z) \tilde{\mu}^{n}(d s, d z) .
$$

We can compute the characteristic function of the random variable $L_{1}^{\alpha, n}$. Indeed, using the exponential formula for Poisson measure,

$$
\begin{aligned}
\mathbb{E}\left[e^{i u L_{1}^{\alpha, n}}\right] & =\exp \left(\int_{\left\{|z| \leq K(a) n^{1 / \alpha}\right\}}\left(e^{i u h_{n}(z)}-1-i u h_{n}(z)\right) \frac{g\left(z n^{-1 / \alpha}\right) \tau\left(z n^{-1 / \alpha}\right)}{|z|^{1+\alpha}} d z\right), \\
& =\exp \left(\int_{\left\{|w| \leq K(a) n^{1 / \alpha}\right\}}\left(e^{i u w}-1-i u w\right) \frac{\tau\left(w n^{-1 / \alpha}\right)}{|w|^{1+\alpha}} d w\right),
\end{aligned}
$$

where in the second line we have used the change of variable $w=h_{n}(z)$ and the relation

$$
\frac{g\left(s_{n}(w) n^{-1 / \alpha}\right) \tau\left(s_{n}(w) n^{-1 / \alpha}\right) s_{n}^{\prime}(w)}{\left|s_{n}(w)\right|^{1+\alpha}}=\frac{\tau\left(w n^{-1 / \alpha}\right)}{|w|^{1+\alpha}},
$$

that can be derived for $w>0$ from the differentiation of the relation $G\left(n^{-1 / \alpha} s_{n}(w)\right)=H\left(n^{-1 / \alpha} w\right)$, and is extended to $w<0$ by symmetry of $g$ and $\tau$. From (4.43) we see that $L_{1}^{\alpha, n}$ has the law of an $\alpha$-stable process whose jumps are truncated with the function $\tau$. Similarly to (4.40) (in the situation $g=1$ ), we deduce

$$
\left|\mathbb{E}\left[h\left(L_{1}^{\alpha}\right)\right]-\mathbb{E}\left[h\left(L_{1}^{\alpha, n}\right)\right]\right| \leq C \frac{\|h\|_{\infty}}{n} .
$$

Theorem 4.2 is a consequence of $(4.40),(4.45)$ and of the following lemma.

Lemma 4.6. Let $h$ be as in Theorem 4.2, then we have,

$$
\left|\mathbb{E}\left[h\left(L_{1}^{\alpha, n}\right)\right]-\mathbb{E}\left[h\left(L_{1}^{n}\right)\right]\right| \leq C \varepsilon_{n}\|h\|_{\infty},
$$

where $\varepsilon_{n}$ is defined in the statement of Theorem 4.1.

Proof. The scheme of the proof follows the same lines as the proof of Theorem 4.1, and is based on the comparison of the representation of the random variables (4.5) and (4.42). Since, in Lemma 4.5, the difference $h_{n}(z)-z$ is only controlled for $|z| \leq \varepsilon n^{1 / \alpha}$ with some $\varepsilon>0$, we need to introduce an additional localization procedure consisting in regularizing $1_{\left\{\mu^{n}\left([0,1] \times\left\{z \in \mathbb{R} ;|z|>\varepsilon n^{1 / \alpha}\right\}\right)=0\right\}}$. Let $\mathcal{I}$ be a smooth function defined on $\mathbb{R}$, and with values in $[0,1]$, such that $\mathcal{I}(x)=1$ for $x \leq 1 / 2$, and $\mathcal{I}(x)=0$ for $x \geq 1$. We denote by $\xi$ a smooth function on $\mathbb{R}$, with values in $[0,1]$ and such that $\xi(z)=0$ for $|z| \leq 1 / 2$ and $\xi(z)=1$ for $|z| \geq 1$, and we set

$$
\begin{aligned}
V^{n}=\int_{0}^{1} \int_{\mathbb{R}} \xi\left(\frac{z}{\varepsilon n^{1 / \alpha}}\right) \mu^{n}(d s, d z)= & \int_{0}^{1} \int_{\left\{\frac{1}{2} \varepsilon n^{1 / \alpha} \leq|z| \leq \varepsilon n^{1 / \alpha}\right\}} \xi\left(\frac{z}{\varepsilon n^{1 / \alpha}}\right) \mu^{n}(d s, d z) \\
& +\int_{0}^{1} \int_{\left\{|z|>\varepsilon n^{1 / \alpha}\right\}} \mu^{n}(d s, d z),
\end{aligned}
$$


From the construction, $W^{n}$ is a Malliavin differentiable random variable such that $W^{n} \neq 0$ implies $\mu^{n}([0,1] \times$ $\left.\left\{z \in \mathbb{R} ;|z|>\varepsilon n^{1 / \alpha}\right\}\right)=0$, and one can show that $P\left(W^{n} \neq 1\right)=O\left(n^{-1}\right)$. From the latter, it is clear that the proof of the lemma reduces in proving the upper bound

$$
\left|\mathbb{E}\left[h\left(L_{1}^{\alpha, n}\right) W^{n}\right]-\mathbb{E}\left[h\left(L_{1}^{n}\right) W^{n}\right]\right| \leq C \varepsilon_{n}\|h\|_{\infty} .
$$

Using a regularizing sequence as in the proof of Theorem 4.1, we can assume that $h$ is $\mathcal{C}^{1}$ with bounded derivative. Then by the integration by part formula (4.21), we can write $\mathbb{E}\left[h\left(L_{1}^{n}\right) W^{n}\right]=\mathbb{E}\left[H\left(L_{1}^{n}\right) \mathcal{H}_{L_{1}^{n}}\left(W^{n}\right)\right]$ where $H$ is some primitive function of $h$ and the Malliavin weight can be written, using (4.22) and the chain rule property of the operator $\Gamma$,

$$
\mathcal{H}_{L_{1}^{n}}\left(W^{n}\right)=W^{n} \mathcal{H}_{L_{1}^{n}}(1)-\frac{\Gamma\left(W^{n}, L_{1}^{n}\right)}{\Gamma\left(L_{1}^{n}, L_{1}^{n}\right)} .
$$

Using the triangle inequality, we are now left to find upper bounds for the following two terms

$$
\begin{aligned}
\widetilde{T}_{1} & :=\left|\mathbb{E}\left[h\left(L_{1}^{\alpha, n}\right) W^{n}\right]-\mathbb{E}\left[H\left(L_{1}^{\alpha, n}\right) \mathcal{H}_{L_{1}^{n}}\left(W^{n}\right)\right]\right|, \\
\widetilde{T}_{2} & :=\left|\mathbb{E}\left[H\left(L_{1}^{\alpha, n}\right) \mathcal{H}_{L_{1}^{n}}\left(W^{n}\right)\right]-\mathbb{E}\left[H\left(L_{1}^{n}\right) \mathcal{H}_{L_{1}^{n}}\left(W^{n}\right)\right]\right| .
\end{aligned}
$$

Bound for $\widetilde{T}_{2}$

Using (4.48) and the Lipschitz property of the function $H$, we have

$$
\widetilde{T}_{2} \leq\|h\|_{\infty} \mathbb{E}\left[\left|L_{1}^{\alpha, n}-L_{1}^{n}\right|\left|\mathcal{H}_{L_{1}^{n}}(1)\right| W^{n}\right]+\|h\|_{\infty} \mathbb{E}\left[\left|L_{1}^{\alpha, n}-L_{1}^{n}\right|\left|\frac{\Gamma\left(W^{n}, L_{1}^{n}\right)}{\Gamma\left(L_{1}^{n}, L_{1}^{n}\right)}\right|\right] .
$$

We focus on the first expectation appearing in the right-hand side of (4.51). Using (4.5) and (4.42), we have

$$
\begin{array}{r}
\mathbb{E}\left[\left|L_{1}^{\alpha, n}-L_{1}^{n}\right|\left|\mathcal{H}_{L_{1}^{n}}(1)\right| W^{n}\right]=\mathbb{E}\left[\left|\int_{0}^{1} \int_{\mathbb{R}}\left(h_{n}(z)-z\right) \tilde{\mu}^{n}(d s, d z)\right|\left|\mathcal{H}_{L_{1}^{n}}(1)\right| W^{n}\right] \\
\leq \mathbb{E}\left[\left|\int_{0}^{1} \int_{\{|z| \leq 1\}}\left(h_{n}(z)-z\right) \tilde{\mu}^{n}(d s, d z)\right| \mid \mathcal{H}_{\left.L_{1}^{n}(1) \mid W^{n}\right]}\right] \\
+\mathbb{E}\left[\left|\int_{0}^{1} \int_{\left\{1<|z| \leq \varepsilon n^{1 / \alpha}\right\}}\left(h_{n}(z)-z\right) \mu^{n}(d s, d z)\right|\left|\mathcal{H}_{L_{1}^{n}}(1)\right| W^{n}\right],
\end{array}
$$

where we have used that $h_{n}$ is an odd function with the symmetry of the compensator $\bar{\mu}^{n}$, and the fact that on $W^{n} \neq 0$ we have $\mu^{n}\left([0,1] \times\left\{z \in \mathbb{R} ;|z|>\varepsilon n^{1 / \alpha}\right\}\right)=0$. The two terms in the right-hand side of (4.52) are controlled using Lemma 4.51 ). For the sake of shortness, we only give the details of the proof in the case $\alpha \neq 1$. In the case $\alpha=1$, one needs to modify this control with an additional logarithmic term. For the small jumps term, from inequality 2.1 .37 in [13] and Lemma 4.51$)$, we deduce $\mathbb{E} \mid \int_{0}^{1} \int_{\{|z| \leq 1\}}\left(h_{n}(z)-\right.$ $z)\left.\tilde{\mu}^{n}(d z, d s)\right|^{p}<C_{p}\left(n^{-1 / \alpha}+n^{-1}\right)^{p}$, for any $p \geq 2$ and using $0 \leq W^{n} \leq 1$ and (4.28) we get from Hölder's inequality

$$
\mathbb{E}\left[\left|\int_{0}^{1} \int_{\{|z| \leq 1\}}\left(h_{n}(z)-z\right) \tilde{\mu}^{n}(s, d z)\right|\left|\mathcal{H}_{L_{1}^{n}}(1)\right| W^{n}\right] \leq C n^{-1 / \alpha}+C n^{-1} .
$$

The large jumps term of (4.52) is upper bounded by

$$
\mathbb{E}\left[\int_{0}^{1} \int_{\left\{1<|z| \leq \varepsilon n^{1 / \alpha}\right\}}\left(\frac{|z|^{\alpha}}{n}+\frac{|z|}{n^{1 / \alpha}}\right) \mu^{n}(d s, d z) \int_{0}^{1} \int_{\left\{1<|z| \leq \varepsilon n^{1 / \alpha}\right\}}|z| \mu^{n}(d s, d z)\left|\mathcal{H}_{L_{1}^{n}}(1)\right| W^{n}\right],
$$


where we have used Lemma 4.51 ), and the basic inequality,

$$
\int_{0}^{1} \int_{\left\{1<|z| \leq \varepsilon n^{1 / \alpha}\right\}}|z|^{\beta} \mu^{n}(d s, d z) \leq \int_{0}^{1} \int_{\left\{1<|z| \leq \varepsilon n^{1 / \alpha}\right\}}|z|^{\beta-1} \mu^{n}(d s, d z) \int_{0}^{1} \int_{\left\{1<|z| \leq \varepsilon n^{1 / \alpha}\right\}}|z| \mu^{n}(d s, d z)
$$

for $\beta \geq 1$. From $\mu^{n}=\tilde{\mu}^{n}+\bar{\mu}^{n}$ and inequality 2.1.36 in [13], one can easily show that for $q \in(1,2)$,

$$
\begin{aligned}
& \mathbb{E}\left[\int_{0}^{1} \int_{\left\{1<|z| \leq \varepsilon n^{1 / \alpha}\right\}}|z| \mu^{n}(d s, d z)\right]^{q} \leq C\left(1+n^{q / \alpha-1}\right), \\
& \mathbb{E}\left[\int_{0}^{1} \int_{\left\{1<|z| \leq \varepsilon n^{1 / \alpha}\right\}}|z|^{\alpha} \mu^{n}(d s, d z)\right]^{q} \leq C n^{q-1} .
\end{aligned}
$$

By Hölder's inequality and (4.29), we deduce that the large jumps term of (4.52) is eventually smaller than $C\left(n^{-1 / \alpha}+n^{-1 / q}\right)$ for any $q \in(1,2)$, and in turn

$$
\mathbb{E}\left[\left|L_{1}^{\alpha, n}-L_{1}^{n}\right|\left|\mathcal{H}_{L_{1}^{n}}(1)\right| W^{n}\right] \leq C \varepsilon_{n} .
$$

Let us now study the second expectation in the right-hand side of (4.51), which can be rewritten, using (4.47) and the chain rule property of the operator $\Gamma$

$$
\mathbb{E}\left[\left|L_{1}^{\alpha, n}-L_{1}^{n}\right|\left|\mathcal{I}^{\prime}\left(V^{n}\right)\right|\left|\frac{\Gamma\left(V^{n}, L_{1}^{n}\right)}{\Gamma\left(L_{1}^{n}, L_{1}^{n}\right)}\right|\right] .
$$

Using (4.46), we get the explicit expression for $\Gamma\left(V^{n}, L_{1}^{n}\right)=\int_{0}^{1} \int_{\mathbb{R}} \rho(z) \xi^{\prime}\left(\frac{z}{\varepsilon n^{1 / \alpha}}\right) \mu^{n}(d s, d z) n^{-1 / \alpha} \varepsilon^{-1}$, from which we deduce $\left|\frac{\Gamma\left(V^{n}, L_{1}^{n}\right)}{\Gamma\left(L_{1}^{n}, L_{1}^{n}\right)}\right| \leq C\left\|\xi^{\prime}\right\|_{\infty} n^{-1 / \alpha}$. Hence, the term (4.53) is smaller than

$$
C n^{-1 / \alpha} \mathbb{E}\left[\left|\int_{0}^{1} \int_{\left\{|z| \leq K(a) n^{1 / \alpha}\right\}}\left(h_{n}(z)-z\right) \widetilde{\mu}^{n}(d s, d z)\right|\left|\mathcal{I}^{\prime}\left(V_{n}\right)\right|\right] .
$$

Remarking that $\mathcal{I}^{\prime}(x)=0$ for $x \geq 1$, we deduce that $\left|\mathcal{I}^{\prime}\left(V^{n}\right)\right| \neq 0$ implies $\mu^{n}([0,1] \times\{z \in \mathbb{R} ;|z|>$ $\left.\left.\varepsilon n^{-1 / \alpha}\right\}\right)=0$. Consequently, (4.53) is upper bounded by

$$
C\left\|\mathcal{I}^{\prime}\right\|_{\infty} n^{-1 / \alpha} \mathbb{E}\left[\left|\int_{0}^{1} \int_{\left\{|z| \leq \varepsilon n^{1 / \alpha}\right\}}\left(h_{n}(z)-z\right) \widetilde{\mu}^{n}(d s, d z)\right|\right],
$$

where we used the symmetry of the compensator $\bar{\mu}^{n}$. Using Lemma 4.5, one can show that

$$
\mathbb{E}\left[\left|\int_{0}^{1} \int_{\left\{|z| \leq \varepsilon n^{1 / \alpha}\right\}}\left(h_{n}(z)-z\right) \widetilde{\mu}^{n}(d s, d z)\right|\right] \leq C n^{1 / \alpha-1},
$$

and deduce that (4.53) is smaller than $C n^{-1}$. This finishes the proof that $\widetilde{T}_{2} \leq C\|h\|_{\infty} \varepsilon_{n}$. Bound for $\widetilde{T}_{1}$

Using (4.17) and (4.22) we can write

$$
\mathcal{H}_{L_{1}^{n}}\left(W^{n}\right)=\frac{-W^{n} L\left(L_{1}^{n}\right)}{\Gamma\left(L_{1}^{n}, L_{1}^{n}\right)}+L\left(\frac{W^{n}}{\Gamma\left(L_{1}^{n}, L_{1}^{n}\right)}\right) L_{1}^{n}-L\left(\frac{L_{1}^{n} W^{n}}{\Gamma\left(L_{1}^{n}, L_{1}^{n}\right)}\right),
$$

and with computations using that $L$ is a self-adjoint operator, as in the proof of Theorem 4.1, we get that

$$
\begin{aligned}
\widetilde{T}_{1} & =\left|\mathbb{E}\left[h\left(L_{1}^{\alpha, n}\right) W^{n}\right]-\mathbb{E}\left[h\left(L_{1}^{\alpha, n}\right) \frac{\Gamma\left(L_{1}^{\alpha, n}, L_{1}^{n}\right)}{\Gamma\left(L_{1}^{n}, L_{1}^{n}\right)} W^{n}\right]\right| \\
& \leq\|h\|_{\infty} \mathbb{E}\left[\left|\frac{\Gamma\left(L_{1}^{n}-L_{1}^{\alpha, n}, L_{1}^{n}\right)}{\Gamma\left(L_{1}^{n}, L_{1}^{n}\right)}\right| W^{n}\right]
\end{aligned}
$$


But $\Gamma\left(L_{1}^{n}-L_{1}^{\alpha, n}, L_{1}^{n}\right)=\int_{0}^{1} \int_{\left\{|z| \leq K(a) n^{1 / \alpha}\right\}} \rho(z)\left(1-h_{n}^{\prime}(z)\right) \mu^{n}(d s, d z)$. Using Lemma 4.52$)$, we deduce that on the event $W^{n} \neq 0$,

$$
\begin{aligned}
\left|\Gamma\left(L_{1}^{n}-L_{1}^{\alpha, n}, L_{1}^{n}\right)\right| \leq & C \int_{0}^{1} \int_{\left\{|z| \leq \varepsilon n^{1 / \alpha}\right\}} \rho(z)\left(\frac{|z|}{n^{1 / \alpha}}+\frac{|z|^{\alpha}}{n}\right) \mu^{n}(d s, d z), \\
\leq & C \int_{0}^{1} \int_{\{|z| \leq 1\}} \rho(z)\left(\frac{|z|}{n^{1 / \alpha}}+\frac{|z|^{\alpha}}{n}\right) \mu^{n}(d s, d z)+ \\
& C \int_{0}^{1} \int_{\left\{1<|z| \leq \varepsilon n^{1 / \alpha}\right\}} \rho(z) \mu^{n}(d s, d z) \int_{0}^{1} \int_{\left\{1<|z| \leq \varepsilon n^{1 / \alpha}\right\}}\left(\frac{|z|}{n^{1 / \alpha}}+\frac{|z|^{\alpha}}{n}\right) \mu^{n}(d s, d z) .
\end{aligned}
$$

From this equation, we deduce that

$$
\begin{aligned}
\widetilde{T}_{1} & \leq C\|h\|_{\infty}\left(n^{-1 / \alpha}+n^{-1}\right)+C\|h\|_{\infty} \mathbb{E} \int_{0}^{1} \int_{\left\{1<|z| \leq \varepsilon n^{1 / \alpha}\right\}}\left(\frac{|z|}{n^{1 / \alpha}}+\frac{|z|^{\alpha}}{n}\right) \mu^{n}(d s, d z), \\
& \leq C\|h\|_{\infty}\left(n^{-1 / \alpha}+\log (n) n^{-1}\right) .
\end{aligned}
$$

This is the required upper bound for $\widetilde{T}_{1}$.

Proof of Lemma 4.5. As $x \mapsto h_{n}(x)$ is an odd function, it is sufficient to study this function on $[0, \infty)$. Recall that for $0<x<K(a) n^{1 / \alpha}, h_{n}(x)=s_{n}^{-1}(x)$ and $s_{n}(x)=n^{1 / \alpha} G^{-1}\left(H\left(n^{-1 / \alpha} x\right)\right)$, where $G$ and $H$ are defined in (4.41). As $\tau(x)=1$ for $|x| \leq K(a) / 2$, we have $H(x)=\alpha^{-1} x^{-\alpha}+\kappa_{1}$ for $0<x<K(a) / 2$, where $\kappa_{1}$ is some constant. Using that $g(x)=1+O(x)$ as $x \rightarrow 0$, we get

$$
G(x)=\int_{x}^{K(a)} \frac{g(z) \tau(z)}{z^{1+\alpha}} d z=\left\{\begin{array}{l}
\alpha^{-1} x^{-\alpha}+\kappa_{2}+O\left(x^{1-\alpha}\right), \quad \text { if } \alpha \neq 1 \\
\alpha^{-1} x^{-\alpha}+O(|\log (x)|), \quad \text { if } \alpha=1,
\end{array}\right.
$$

where $\kappa_{2}$ is some constant. Then, by elementary computations we deduce that if $u \in(0, \infty)$ is large enough,

$$
\left|G^{-1}(u)-(\alpha u)^{-1 / \alpha}\right| \leq\left\{\begin{array}{l}
C u^{-2 / \alpha}+C u^{-1-1 / \alpha}, \quad \text { if } \alpha \neq 1 \\
C u^{-2}|\log (u)|, \quad \text { if } \alpha=1 .
\end{array}\right.
$$

From the expression $H(x)=\alpha^{-1} x^{-\alpha}+\kappa_{1}$ and (4.54), it comes, for $x / n^{1 / \alpha}$ small enough,

$$
\left|s_{n}(x)-x\right|=\left|n^{1 / \alpha} G^{-1}\left(H\left(n^{-1 / \alpha} x\right)\right)-x\right| \leq\left\{\begin{array}{l}
C \frac{x^{2}}{n^{1 / \alpha}}+C \frac{x^{1+\alpha}}{n}, \quad \text { if } \alpha \neq 1 \\
C \frac{x^{2}}{n}\left|\log \left(\frac{x}{n}\right)\right| \text { if } \alpha=1 .
\end{array}\right.
$$

Now, the first part of the lemma follows from $h_{n}=s_{n}^{-1}$.

For the second part we use (4.44) to get, if $x / n^{1 / \alpha}$ is small enough,

$$
s_{n}^{\prime}(x)=\left(\frac{s_{n}(x)}{x}\right)^{1+\alpha} \frac{1}{g\left(n^{-1 / \alpha} s_{n}(x)\right)} .
$$

From (4.55) and $\frac{1}{g\left(n^{-1 / \alpha} s_{n}(x)\right)}=1+O\left(s_{n}(x) / n^{1 / \alpha}\right)=1+O\left(x / n^{1 / \alpha}\right)$, we deduce the second part of the lemma.

\section{Proof of Theorems 3.1 and 3.2}

\subsection{Proof of Theorem 3.1}

Before proceeding to the proof of Theorem 3.1, we first recall the following useful result to prove convergence in probability of triangular arrays (see [13]). 
Let $\left(\zeta_{i}^{n}\right)$ be a triangular array such that $\zeta_{i}^{n}$ is $\mathcal{F}_{\frac{i}{n}}$-measurable then the two following conditions imply the convergence in probability $\sum_{i=1}^{n} \zeta_{i}^{n} \rightarrow 0$ :

$$
\begin{aligned}
& \sum_{i=1}^{n}\left|\mathbb{E}_{\mid \mathcal{F}_{\frac{i-1}{n}}} \zeta_{i}^{n}\right| \rightarrow 0 \quad \text { in probability, } \\
& \sum_{i=1}^{n} \mathbb{E}_{\mid \mathcal{F}_{\frac{i-1}{n}}}\left|\zeta_{i}^{n}\right|^{2} \rightarrow 0 \quad \text { in probability. }
\end{aligned}
$$

To prove Theorem 3.1, the idea is to replace $z_{n}$ defined by (3.4), for $(\theta, \sigma) \in V_{n}^{(\eta)}\left(\theta_{0}, \sigma_{0}\right)$, by the normalized increment $n^{1 / \alpha} \Delta L_{i}=n^{1 / \alpha}\left(L_{\frac{i}{n}}-L_{\frac{i-1}{n}}\right)$. This approximation is justify by the next lemma which is an extension of Lemma 4.2 given in Section 4.1.

Lemma 5.1. Assuming $H 1$ and H2(a), there exists $p, q>0$ such that

$$
\forall \varepsilon>0, \mathbb{P}_{\mid \mathcal{F}_{\frac{i-1}{n}}}\left(\sup _{(\theta, \sigma) \in V_{n}^{(\eta)}\left(\theta_{0}, \sigma_{0}\right)}\left|z_{n}\left(X_{\frac{i-1}{n}}, X_{\frac{i}{n}}, \theta, \sigma\right)-n^{1 / \alpha} \Delta L_{i}\right|>\varepsilon\right) \leq C(\varepsilon)\left(1+\left|X_{\frac{i-1}{n}}\right|^{p}\right) / n^{q},
$$

where $C(\varepsilon)$ is a positive constant and $\Delta L_{i}=L_{\frac{i}{n}}-L_{\frac{i-1}{n}}$.

Proof. We have the decomposition

$$
\begin{array}{r}
z_{n}\left(X_{\frac{i-1}{n}}, X_{\frac{i}{n}}, \theta, \sigma\right)=\frac{a\left(X_{\frac{i-1}{n}}, \sigma_{0}\right)\left(z_{n}\left(X_{\frac{i-1}{n}}, X_{\frac{i}{n}}, \theta_{0}, \sigma_{0}\right)-n^{1 / \alpha} \Delta L_{i}\right)}{a\left(X_{\frac{i-1}{n}}, \sigma\right)}+\frac{a\left(X_{\frac{i-1}{n}}, \sigma_{0}\right)}{a\left(X_{\frac{i-1}{n}}, \sigma\right)} n^{1 / \alpha} \Delta L_{i} \\
+\frac{n^{1 / \alpha}}{a\left(X_{\frac{i-1}{n}}, \sigma\right)}\left(\xi_{i-1}\left(\theta_{0}\right)-\xi_{i-1}(\theta)\right) .
\end{array}
$$

From $\mathrm{H} 1$ we have for $t \in[0,1 / n]$ and $\theta \in V\left(\theta_{0}\right)$

$$
\left|\xi_{t}^{x}(\theta)-\xi_{t}^{x}\left(\theta_{0}\right)\right| \leq\left\|b^{\prime}\right\|_{\infty} \int_{0}^{t}\left|\xi_{s}^{x}(\theta)-\xi_{s}^{x}\left(\theta_{0}\right)\right| d s+\left|\theta-\theta_{0}\right|\left(1+\sup _{t \in[0,1 / n]}\left|\xi_{t}^{x}\left(\theta_{0}\right)\right|^{p}\right) / n,
$$

moreover from Gronwall's Lemma we check easily that

$$
\sup _{t \in[0,1 / n]}\left|\xi_{t}^{x}\left(\theta_{0}\right)\right| \leq C(1+|x|) .
$$

This leads to the bound (using once again Gronwall's Lemma)

$$
\left|\xi_{i-1}\left(\theta_{0}\right)-\xi_{i-1}(\theta)\right| \leq \frac{C}{n}\left|\theta-\theta_{0}\right|\left(1+\left|X_{\frac{i-1}{n}}\right|^{p}\right) .
$$

Now for $(\theta, \sigma) \in V_{n}^{(\eta)}\left(\theta_{0}, \sigma_{0}\right)$, we have $\left|\theta-\theta_{0}\right| \leq \eta / n^{1 / \alpha-1 / 2}$ and $\left|\sigma-\sigma_{0}\right| \leq \eta / n^{1 / 2}$ so from H1 we get that

$$
\left|\frac{n^{1 / \alpha}}{a\left(X_{\frac{i-1}{n}}, \sigma\right)}\left(\xi_{i-1}\left(\theta_{0}\right)-\xi_{i-1}(\theta)\right)\right| \leq C\left(1+\left|X_{\frac{i-1}{n}}\right|^{p}\right) / n^{1 / 2} .
$$

Using once again $\mathrm{H} 1$ and a Taylor expansion, we have for $(\theta, \sigma) \in V_{n}^{(\eta)}\left(\theta_{0}, \sigma_{0}\right)$,

$$
\left|\frac{a\left(X_{\frac{i-1}{n}}, \sigma_{0}\right)}{a\left(X_{\frac{i-1}{n}}, \sigma\right)}-1\right| \leq C\left(1+\left|X_{\frac{i-1}{n}}\right|^{p}\right) / n^{1 / 2} .
$$


This gives the bound

$$
\begin{aligned}
\sup _{(\theta, \sigma) \in V_{n}^{(\eta)}\left(\theta_{0}, \sigma_{0}\right)}\left|z_{n}\left(X_{\frac{i-1}{n}}, X_{\frac{i}{n}}, \theta, \sigma\right)-n^{1 / \alpha} \Delta L_{i}\right| & \leq C\left(1+\left|X_{\frac{i-1}{n}}\right|^{p}\right)\left|z_{n}\left(X_{\frac{i-1}{n}}, X_{\frac{i}{n}}, \theta_{0}, \sigma_{0}\right)-n^{1 / \alpha} \Delta L_{i}\right| \\
& +C\left(1+\left|X_{\frac{i-1}{n}}\right|^{p}\right) \frac{n^{1 / \alpha}\left|\Delta L_{i}\right|}{n^{1 / 2}}+C\left(1+\left|X_{\frac{i-1}{n}}\right|^{p}\right) \frac{1}{n^{1 / 2}} .
\end{aligned}
$$

The Markov property and the result of Lemma 4.2 give for some $p^{\prime}, q>0$

$$
\mathbb{P}_{\mid \mathcal{F}_{\frac{i-1}{n}}}\left(C\left(1+\left|X_{\frac{i-1}{n}}\right|^{p}\right)\left|z_{n}\left(X_{\frac{i-1}{n}}, X_{\frac{i}{n}}, \theta_{0}, \sigma_{0}\right)-n^{1 / \alpha} \Delta L_{i}\right|>\varepsilon\right) \leq C(\varepsilon)\left(1+\left|X_{\frac{i-1}{n}}\right|^{p^{\prime}}\right) / n^{q} .
$$

Moreover from Theorem 2 in Luschgy-Pagès [19], we have $\mathbb{E}\left|n^{1 / \alpha} \Delta L_{i}\right|^{q} \leq C$, for $q<\alpha$ and we deduce that

$$
\mathbb{P}_{\mid \mathcal{F}_{\frac{i-1}{n}}}\left(\left(1+\left|X_{\frac{i-1}{n}}\right|^{p}\right) \frac{n^{1 / \alpha}\left|\Delta L_{i}\right|}{n^{1 / 2}}>\varepsilon\right) \leq C(\varepsilon)\left(1+\left|X_{\frac{i-1}{n}}\right|^{p q}\right) / n^{q / 2} .
$$

This finally leads to the bound (5.3).

We can now proceed to the proof of Theorem 3.1.

Proof of Theorem 3.1.

i) From the triangle inequality, it is sufficient to prove the four following convergences in probability:

$$
\begin{aligned}
& \sup _{(\theta, \sigma) \in V_{n}^{(\eta)}\left(\theta_{0}, \sigma_{0}\right)}\left|\frac{1}{n} \sum_{i=1}^{n}\left(f\left(X_{\frac{i-1}{n}}, \theta, \sigma\right)-f\left(X_{\frac{i-1}{n}}, \theta_{0}, \sigma_{0}\right)\right) h\left(z_{n}\left(X_{\frac{i-1}{n}}, X_{\frac{i}{n}}, \theta, \sigma\right)\right)\right| \rightarrow 0, \\
& \sup _{(\theta, \sigma) \in V_{n}^{(\eta)}\left(\theta_{0}, \sigma_{0}\right)}\left|\frac{1}{n} \sum_{i=1}^{n} f\left(X_{\frac{i-1}{n}}, \theta_{0}, \sigma_{0}\right)\left(h\left(z_{n}\left(X_{\frac{i-1}{n}}, X_{\frac{i}{n}}, \theta, \sigma\right)\right)-h\left(n^{1 / \alpha} \Delta L_{i}\right)\right)\right| \rightarrow 0, \\
& \left|\frac{1}{n} \sum_{i=1}^{n} f\left(X_{\frac{i-1}{n}}, \theta_{0}, \sigma_{0}\right)\left(h\left(n^{1 / \alpha} \Delta L_{i}\right)-\mathbb{E} h\left(L_{1}^{\alpha}\right)\right)\right| \rightarrow 0 \\
& \left|\frac{1}{n} \sum_{i=1}^{n} f\left(X_{\frac{i-1}{n}}, \theta_{0}, \sigma_{0}\right)-\int_{0}^{1} f\left(X_{s}, \theta_{0}, \sigma_{0}\right) d s\right| \rightarrow 0
\end{aligned}
$$

The last point (5.7) is the convergence of a Riemann sum since $t \mapsto X_{t}$ is càdlàg and $f$ is continuous.

Since $h$ is bounded and $\sup _{(\theta, \sigma) \in V_{\theta_{0}} \times V_{\sigma_{0}}}\left(\left|\partial_{\theta} f(x, \theta, \sigma)\right|+\left|\partial_{\sigma} f(x, \theta, \sigma)\right|\right) \leq C\left(1+|x|^{p}\right)$, we get easily (5.4) from a Taylor expansion and using $\left|\theta-\theta_{0}\right| \leq \eta / n^{1 / \alpha-1 / 2}$ and $\left|\sigma-\sigma_{0}\right| \leq \eta / n^{1 / 2}$, for $(\theta, \sigma) \in V_{n}^{(\eta)}\left(\theta_{0}, \sigma_{0}\right)$.

To prove (5.5), we introduce the truncation $1_{\left\{\sup _{(\theta, \sigma) \in V_{n}^{(\eta)}\left(\theta_{0}, \sigma_{0}\right)}\left|z_{n}\left(X_{\frac{i-1}{n}}, X_{\frac{i}{n}}, \theta, \sigma\right)-n^{1 / \alpha} \Delta L_{i}\right| \leq \varepsilon\right\}}$ for $\varepsilon>0$, and since $h$ and $h^{\prime}$ are bounded we get

$$
\begin{array}{r}
\sup _{(\theta, \sigma) \in V_{n}^{(\eta)}\left(\theta_{0}, \sigma_{0}\right)}\left|h\left(z_{n}\left(X_{\frac{i-1}{n}}, X_{\frac{i}{n}}, \theta, \sigma\right)\right)-h\left(n^{1 / \alpha} \Delta L_{i}\right)\right| \leq \varepsilon\left\|h^{\prime}\right\|_{\infty}+ \\
2\|h\|_{\infty} 1_{\left\{\sup _{(\theta, \sigma) \in V_{n}^{(\eta)}\left(\theta_{0}, \sigma_{0}\right)} \mid z_{n}\left(X_{\left.\left.\frac{i-1}{n}, X_{\frac{i}{n}}, \theta, \sigma\right)-n^{1 / \alpha} \Delta L_{i} \mid>\varepsilon\right\}} .\right.\right.} .
\end{array}
$$

This yields

$$
\begin{array}{r}
\sup _{(\theta, \sigma) \in V_{n}^{(\eta)}\left(\theta_{0}, \sigma_{0}\right)}\left|\frac{1}{n} \sum_{i=1}^{n} f\left(X_{\frac{i-1}{n}}, \theta_{0}, \sigma_{0}\right)\left(h\left(z_{n}\left(X_{\frac{i-1}{n}}, X_{\frac{i}{n}}, \theta, \sigma\right)\right)-h\left(n^{1 / \alpha} \Delta L_{i}\right)\right)\right| \leq \varepsilon C\left(1+\sup _{s \in[0,1]}\left|X_{s}\right|^{p}\right)\left\|h^{\prime}\right\|_{\infty} \\
+C\|h\|_{\infty} \frac{1}{n} \sum_{i=1}^{n}\left(1+\left|X_{\frac{i-1}{n}}\right|^{p}\right) 1_{\left\{\sup _{(\theta, \sigma) \in V_{n}^{(\eta)}\left(\theta_{0}, \sigma_{0}\right)}\left|z_{n}\left(X_{\frac{i-1}{n}}, X_{\frac{i}{n}}, \theta, \sigma\right)-n^{1 / \alpha} \Delta L_{i}\right|>\varepsilon\right\} .} .
\end{array}
$$




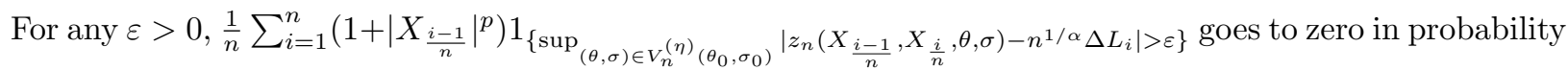
(we check easily (5.1) and (5.2) from Lemma 5.1) and we deduce (5.5) letting $\varepsilon$ go to zero.

The proof of (5.6) is established by checking (5.1) and (5.2) with

$$
\zeta_{i}^{n}=\frac{1}{n} f\left(X_{\frac{i-1}{n}}, \theta_{0}, \sigma_{0}\right)\left(h\left(n^{1 / \alpha} \Delta L_{i}\right)-\mathbb{E} h\left(L_{1}^{\alpha}\right)\right) .
$$

From Theorem 4.2 we get (5.1) and the boudedness of $h$ implies immediately (5.2).

ii) As mentioned before, only the case $\alpha>1$ requires a proof.

We first remark that we just have to prove the convergence in probability, for any $K>0$ fixed

$$
\sup _{(\theta, \sigma) \in V_{n}^{(\eta)}\left(\theta_{0}, \sigma_{0}\right)}\left|\frac{1}{n^{1 / \alpha}} \sum_{i=1}^{n} f\left(X_{\frac{i-1}{n}}, \theta, \sigma\right) 1_{\left\{\left|X_{\frac{i-1}{n}}\right| \leq K\right\}} h\left(z_{n}\left(X_{\frac{i-1}{n}}, X_{\frac{i}{n}}, \theta, \sigma\right)\right)\right| \rightarrow 0 .
$$

Indeed, $1_{\left\{\left|X_{\frac{i-1}{n}}\right|>K\right\}} \leq 1_{\left\{\sup _{t \in[0,1]}\left|X_{t}\right|>K\right\}}$ and since $\mathbb{P}\left(\sup _{t \in[0,1]}\left|X_{t}\right|>K\right)$ goes to zero as $K$ goes to infinity, we deduce ii) from (5.8) letting successively $n$ and $K$ go to infinity.

The proof of (5.8) is obtained by establishing the three following convergences in probability :

$$
\begin{gathered}
\sup _{(\theta, \sigma) \in V_{n}^{(\eta)}\left(\theta_{0}, \sigma_{0}\right)} \mid \frac{1}{n^{1 / \alpha}} \sum_{i=1}^{n} f\left(X_{\frac{i-1}{n}}, \theta, \sigma\right) 1_{\left\{\left|X_{\frac{i-1}{n}}\right| \leq K\right\}}\left[h\left(z_{n}\left(X_{\frac{i-1}{n}}, X_{\frac{i}{n}}, \theta, \sigma\right)\right)-\right. \\
\left.h\left(z_{n}\left(X_{\frac{i-1}{n}}, X_{\frac{i}{n}}, \theta_{0}, \sigma_{0}\right)\right)\right] \mid \rightarrow 0, \\
\sup _{(\theta, \sigma) \in V_{n}^{(\eta)}\left(\theta_{0}, \sigma_{0}\right)} \mid \frac{1}{n^{1 / \alpha}} \sum_{i=1}^{n} f\left(X_{\frac{i-1}{n}}, \theta, \sigma\right) 1_{\left\{\left|X_{\frac{i-1}{n}}\right| \leq K\right\}}\left[h\left(z_{n}\left(X_{\frac{i-1}{n}}, X_{\frac{i}{n}}, \theta_{0}, \sigma_{0}\right)\right)-\right. \\
\left.\mathbb{E}_{\mid \mathcal{F} \frac{i-1}{n}} h\left(z_{n}\left(X_{\frac{i-1}{n}}, X_{\frac{i}{n}}, \theta_{0}, \sigma_{0}\right)\right)\right] \mid \rightarrow 0, \\
\sup _{(\theta, \sigma) \in V_{n}^{(\eta)}\left(\theta_{0}, \sigma_{0}\right)}\left|\frac{1}{n^{1 / \alpha}} \sum_{i=1}^{n} f\left(X_{\frac{i-1}{n}}, \theta, \sigma\right) 1_{\left\{\left|X_{\frac{i-1}{n}}\right| \leq K\right\}} \mathbb{E}_{\mid \mathcal{F}_{\frac{i-1}{n}}} h\left(z_{n}\left(X_{\frac{i-1}{n}}, X_{\frac{i}{n}}, \theta_{0}, \sigma_{0}\right)\right)\right| \rightarrow 0 .
\end{gathered}
$$

Considering first (5.9), we have

$$
z_{n}\left(X_{\frac{i-1}{n}}, X_{\frac{i}{n}}, \theta, \sigma\right)=\frac{a\left(X_{\frac{i-1}{n}}, \sigma_{0}\right)}{a\left(X_{\frac{i-1}{n}}, \sigma\right)} z_{n}\left(X_{\frac{i-1}{n}}, X_{\frac{i}{n}}, \theta_{0}, \sigma_{0}\right)+\frac{n^{1 / \alpha}}{a\left(X_{\frac{i-1}{n}}, \sigma\right)}\left(\xi_{i-1}\left(\theta_{0}\right)-\xi_{i-1}(\theta)\right) .
$$

Now, we have the bounds (this has been established in the proof of Lemma 5.1)

$$
\begin{gathered}
\sup _{(\theta, \sigma) \in V_{n}^{(\eta)}\left(\theta_{0}, \sigma_{0}\right)}\left|\frac{n^{1 / \alpha}}{a\left(X_{\frac{i-1}{n}}, \sigma\right)}\left(\xi_{i-1}\left(\theta_{0}\right)-\xi_{i-1}(\theta)\right)\right| \leq C\left(1+\left|X_{\frac{i-1}{n}}\right|^{p}\right) / n^{1 / 2}, \\
\sup _{(\theta, \sigma) \in V_{n}^{(\eta)}\left(\theta_{0}, \sigma_{0}\right)}\left|\frac{a\left(X_{\frac{i-1}{n}}, \sigma_{0}\right)}{a\left(X_{\frac{i-1}{n}}, \sigma\right)}-1\right| \leq C\left(1+\left|X_{\frac{i-1}{n}}\right|^{p}\right) / n^{1 / 2} .
\end{gathered}
$$

This leads to

$$
\sup _{(\theta, \sigma) \in V_{n}^{(\eta)}\left(\theta_{0}, \sigma_{0}\right)}\left|z_{n}\left(X_{\frac{i-1}{n}}, X_{\frac{i}{n}}, \theta, \sigma\right)-z_{n}\left(X_{\frac{i-1}{n}}, X_{\frac{i}{n}}, \theta_{0}, \sigma_{0}\right)\right| \leq C\left(1+\left|X_{\frac{i-1}{n}}\right|^{p}\right)\left(\frac{\left|z_{n}\left(X_{\frac{i-1}{n}}, X_{\frac{i}{n}}, \theta_{0}, \sigma_{0}\right)\right|}{n^{1 / 2}}+\frac{1}{n^{1 / 2}}\right),
$$

and finally adding and subtracting $n^{1 / \alpha} \Delta L_{i}$

$$
\begin{aligned}
\sup _{(\theta, \sigma) \in V_{n}^{(\eta)}\left(\theta_{0}, \sigma_{0}\right)}\left|z_{n}\left(X_{\frac{i-1}{n}}, X_{\frac{i}{n}}, \theta, \sigma\right)-z_{n}\left(X_{\frac{i-1}{n}}, X_{\frac{i}{n}}, \theta_{0}, \sigma_{0}\right)\right| \\
\leq C\left(1+\left|X_{\frac{i-1}{n}}\right|^{p}\right) \frac{\left|z_{n}\left(X_{\frac{i-1}{n}}, X_{\frac{i}{n}}, \theta_{0}, \sigma_{0}\right)-n^{1 / \alpha} \Delta L_{i}\right|+n^{1 / \alpha}\left|\Delta L_{i}\right|+1}{n^{1 / 2}} .
\end{aligned}
$$


Introducing the truncation $1_{\left\{\left|z_{n}\left(X_{\frac{i-1}{n}}, X_{\frac{i}{n}}, \theta_{0}, \sigma_{0}\right)-n^{1 / \alpha} \Delta L_{i}\right| \leq \varepsilon\right\}}$ for $\varepsilon>0$, we deduce that

$$
\begin{aligned}
\sup _{(\theta, \sigma) \in V_{n}^{(\eta)}\left(\theta_{0}, \sigma_{0}\right)}\left|f\left(X_{\frac{i-1}{n}}, \theta, \sigma\right) 1_{\left\{\left|X_{\frac{i-1}{n}}\right| \leq K\right\}}\left(h\left(z_{n}\left(X_{\frac{i-1}{n}}, X_{\frac{i}{n}}, \theta, \sigma\right)\right)-h\left(z_{n}\left(X_{\frac{i-1}{n}}, X_{\frac{i}{n}}, \theta_{0}, \sigma_{0}\right)\right)\right)\right| \leq \\
C_{K}\left(\left\|h^{\prime}\right\|_{\infty} \frac{\left(\varepsilon+1+n^{1 / \alpha}\left|\Delta L_{i}\right|\right)}{n^{1 / 2}}+\|h\|_{\infty} 1_{\left\{\left|z_{n}\left(X_{\frac{i-1}{n}}, X_{\frac{i}{n}}, \theta_{0}, \sigma_{0}\right)-n^{1 / \alpha} \Delta L_{i}\right|>\varepsilon\right\}}\right) .
\end{aligned}
$$

Observing that $1 / \alpha+1 / 2>1$, to prove (5.9) it remains to check the two convergences in probability

$$
\frac{1}{n^{1 / \alpha}} \sum_{i=1}^{n} \frac{n^{1 / \alpha}\left|\Delta L_{i}\right|}{n^{1 / 2}} \rightarrow 0, \quad \frac{1}{n^{1 / \alpha}} \sum_{i=1}^{n} 1_{\left\{\left|z_{n}\left(X_{\frac{i-1}{n}}, X_{\frac{i}{n}}, \theta_{0}, \sigma_{0}\right)-n^{1 / \alpha} \Delta L_{i}\right|>\varepsilon\right\}} \rightarrow 0 .
$$

Since $\alpha>1$, Theorem 2 in [19] gives $\mathbb{E} n^{1 / \alpha}\left|\Delta L_{i}\right| \leq C$ and we deduce $\mathbb{E}\left[\frac{1}{n^{1 / \alpha}} \sum_{i=1}^{n} \frac{n^{1 / \alpha}\left|\Delta L_{i}\right|}{n^{1 / 2}}\right] \rightarrow 0$. Moreover using the result of Lemma 4.2 , we deduce $\mathbb{E} 1_{\left\{\left|z_{n}\left(X_{\frac{i-1}{n}}, X_{\frac{i}{n}}, \theta_{0}, \sigma_{0}\right)-n^{1 / \alpha} \Delta L_{i}\right|>\varepsilon\right\}} \leq C_{K} C(\varepsilon) / n^{1-\delta}$ for any $\delta \in$ $(0,1)$ and this permits to obtain the second convergence. This achieves the proof of $(5.9)$.

Turning to (5.11), since $\mathbb{E} h\left(L_{1}^{\alpha}\right)=0$, we deduce from Theorem 4.2

$$
\left|\mathbb{E}_{\mid \mathcal{F}_{\frac{i-1}{n}}} h\left(n^{1 / \alpha} \Delta L_{i}\right)\right| \leq C \varepsilon_{n}
$$

moreover from Theorem 4.1, we get

$$
\left|\mathbb{E}_{\mid \mathcal{F}_{\frac{i-1}{n}}}\left(h\left(z_{n}\left(X_{\frac{i-1}{n}}, X_{\frac{i}{n}}, \theta_{0}, \sigma_{0}\right)\right)-h\left(n^{1 / \alpha} \Delta L_{i}\right)\right)\right| \leq C\left(1+\left|X_{\frac{i-1}{n}}\right|\right) \varepsilon_{n},
$$

with $\sqrt{n} \varepsilon_{n} \rightarrow 0$. This permits to conclude that (5.11) holds.

It remains to prove the uniform convergence of the martingale part (5.10). For any $(\theta, \sigma) \in V_{\theta_{0}} \times V_{\sigma_{0}}$ the convergence in probability

$$
\frac{1}{n^{1 / \alpha}} \sum_{i=1}^{n} f\left(X_{\frac{i-1}{n}}, \theta, \sigma\right) 1_{\left\{\left|X_{\frac{i-1}{n}}\right| \leq K\right\}}\left(h\left(z_{n}\left(X_{\frac{i-1}{n}}, X_{\frac{i}{n}}, \theta_{0}, \sigma_{0}\right)\right)-\mathbb{E}_{\mid \mathcal{F}_{\frac{i-1}{n}}} h\left(z_{n}\left(X_{\frac{i-1}{n}}, X_{\frac{i}{n}}, \theta_{0}, \sigma_{0}\right)\right)\right) \rightarrow 0,
$$

is immediate (we check easily $(5.2)$ since $2 / \alpha>1$ ). To prove the uniform convergence we use a tightness criteria (see for example the Appendix of [10]). Denoting

$$
M_{n}(\theta, \sigma)=\frac{1}{n^{1 / \alpha}} \sum_{i=1}^{n} f\left(X_{\frac{i-1}{n}}, \theta, \sigma\right) 1_{\left\{\left|X_{\frac{i-1}{n}}\right| \leq K\right\}}\left(h\left(z_{n}\left(X_{\frac{i-1}{n}}, X_{\frac{i}{n}}, \theta_{0}, \sigma_{0}\right)\right)-\mathbb{E}_{\mid \mathcal{F}_{\frac{i-1}{n}}} h\left(z_{n}\left(X_{\frac{i-1}{n}}, X_{\frac{i}{n}}, \theta_{0}, \sigma_{0}\right)\right)\right),
$$

it is sufficient to check, for $\left(\theta_{i}, \sigma_{i}\right) \in V_{\theta_{0}} \times V_{\sigma_{0}}, i=1,2$,

$$
\sup _{n} \mathbb{E}\left|M_{n}\left(\theta_{1}, \sigma_{1}\right)-M_{n}\left(\theta_{2}, \sigma_{2}\right)\right|^{4} \leq C\left\|\left(\theta_{1}, \sigma_{1}\right)^{T}-\left(\theta_{2}, \sigma_{2}\right)^{T}\right\|^{4} .
$$

From Burkholder inequality for discrete martingale (see [22]) :

$$
\mathbb{E}\left|M_{n}\left(\theta_{1}, \sigma_{1}\right)-M_{n}\left(\theta_{2}, \sigma_{2}\right)\right|^{4} \leq \frac{C}{n^{4 / \alpha}} \mathbb{E}\left(\sum_{i=1}^{n}\left|m_{i}\left(\theta_{1}, \sigma_{1}\right)-m_{i}\left(\theta_{2}, \sigma_{2}\right)\right|^{2}\right)^{2},
$$

where $m_{i}(\theta, \sigma)=f\left(X_{\frac{i-1}{n}}, \theta, \sigma\right) 1_{\left\{\left|X_{\frac{i-1}{n}}\right| \leq K\right\}}\left(h\left(z_{n}\left(X_{\frac{i-1}{n}}, X_{\frac{i}{n}}, \theta_{0}, \sigma_{0}\right)\right)-\mathbb{E}_{\mid \mathcal{F}_{\frac{i-1}{n}}} h\left(z_{n}\left(X_{\frac{i-1}{n}}, X_{\frac{i}{n}}, \theta_{0}, \sigma_{0}\right)\right)\right)$, and from Cauchy-Schwarz inequality

$$
\mathbb{E}\left|M_{n}\left(\theta_{1}, \sigma_{1}\right)-M_{n}\left(\theta_{2}, \sigma_{2}\right)\right|^{4} \leq \frac{C}{n^{4 / \alpha}} n \mathbb{E} \sum_{i=1}^{n}\left|m_{i}\left(\theta_{1}, \sigma_{1}\right)-m_{i}\left(\theta_{2}, \sigma_{2}\right)\right|^{4} .
$$


This gives from a first order Taylor expansion of $f$

$$
\mathbb{E}\left|M_{n}\left(\theta_{1}, \sigma_{1}\right)-M_{n}\left(\theta_{2}, \sigma_{2}\right)\right|^{4} \leq \frac{C_{K}}{n^{4 / \alpha}} n^{2}\|h\|_{\infty}\left\|\left(\theta_{1}, \sigma_{1}\right)^{T}-\left(\theta_{2}, \sigma_{2}\right)^{T}\right\|^{4} .
$$

Since $n^{2} / n^{4 / \alpha} \rightarrow 0$, the result is established.

\subsection{Proof of Theorem 3.2 and Corollary 3.1}

\section{Proof of Theorem 3.2.}

We will prove the stable convergence in law with respect to $\sigma\left(L_{s}, s \leq 1\right)$ of the process

$$
\Gamma_{t}^{n}=\frac{1}{n^{1 / 2}} \sum_{i=1}^{[n t]}\left(\begin{array}{c}
f_{1}\left(X_{\frac{i-1}{n}}\right) h_{1}\left(n^{1 / \alpha} \Delta L_{i}\right) \\
f_{2}\left(X_{\frac{i-1}{n}}\right) h_{2}\left(n^{1 / \alpha} \Delta L_{i}\right)
\end{array}\right), \quad t \in[0,1],
$$

in $\mathbb{D}\left([0,1], \mathbb{R}^{2}\right)$ equipped with the Skorokhod topology. To this end we introduce the processes

$$
\begin{gathered}
\bar{L}_{t}^{n}=\sum_{i=1}^{[n t]} \Delta L_{i}, \quad t \in[0,1], \\
\Gamma_{t}^{\prime n}=\frac{1}{n^{1 / 2}} \sum_{i=1}^{[n t]}\left(\begin{array}{c}
h_{1}\left(n^{1 / \alpha} \Delta L_{i}\right) \\
h_{2}\left(n^{1 / \alpha} \Delta L_{i}\right)
\end{array}\right), \quad t \in[0,1] .
\end{gathered}
$$

The process $\left(\bar{L}_{t}^{n}\right)_{t}$ converges in probability to $\left(L_{t}\right)_{t}$ for the Skorokhod topology and according to Lemma 2.8 in [12], if $\left(\bar{L}_{1}^{n}, \Gamma_{1}^{\prime n}\right)$ converges in law to $\left(L_{1}, \gamma^{\prime}\right)$ where $\gamma^{\prime}$ is a Gaussian variable independent of $L_{1}$ with variance

$$
\Sigma^{\prime}=\left(\begin{array}{cc}
\mathbb{E} h_{1}^{2}\left(L_{1}^{\alpha}\right) & 0 \\
0 & \mathbb{E} h_{2}^{2}\left(L_{1}^{\alpha}\right)
\end{array}\right)
$$

then there exists a two-dimensional standard Brownian motion $\left(B_{t}\right)=\left(B_{t}^{1}, B_{t}^{2}\right)$ independent of $\left(L_{t}\right)$ such that the processes $\left(\bar{L}^{n}, \Gamma^{n}, \Gamma^{\prime n}\right)$ converge in law to $\left(L, \Gamma,\left(\Sigma^{\prime}\right)^{1 / 2} B\right)$, where

$$
\Gamma_{t}=\int_{0}^{t}\left(\begin{array}{cc}
f_{1}\left(X_{s}\right) & 0 \\
0 & f_{2}\left(X_{s}\right)
\end{array}\right)\left(\Sigma^{\prime}\right)^{1 / 2} d B_{s}
$$

This result implies the stable convergence stated in Theorem 3.2.

To study the convergence in law of $\left(\bar{L}_{1}^{n}, \Gamma_{1}^{\prime n}\right)$, we denote by $\Phi_{n}$ the characteristic function of $\left(\bar{L}_{1}^{n}, \Gamma_{1}^{\prime n}\right)$, and by $\phi_{n}$ the characteristic function of the $\left(L_{1 / n}, \frac{1}{n^{1 / 2}} h_{1}\left(n^{1 / \alpha} L_{1 / n}\right), \frac{1}{n^{1 / 2}} h_{2}\left(n^{1 / \alpha} L_{1 / n}\right)\right)$. Then we have

$$
\log \Phi_{n}=n \log \phi_{n},
$$

and we just have to study the asymptotic behavior of $\phi_{n}$. By definition

$$
\phi_{n}(u, v, w)=\mathbb{E} e^{i u L_{1 / n}+i \frac{v}{n^{1 / 2}} h_{1}\left(n^{1 / \alpha} L_{1 / n}\right)+i \frac{w}{n^{1 / 2}} h_{2}\left(n^{1 / \alpha} L_{1 / n}\right)} .
$$

A Taylor expansion of the exponential function gives :

$$
\begin{array}{r}
e^{i \frac{v}{n^{1 / 2}} h_{1}\left(n^{1 / \alpha} L_{1 / n}\right)+i \frac{w}{n^{1 / 2}} h_{2}\left(n^{1 / \alpha} L_{1 / n}\right)}=1+i \frac{v}{n^{1 / 2}} h_{1}\left(n^{1 / \alpha} L_{1 / n}\right)+i \frac{w}{n^{1 / 2}} h_{2}\left(n^{1 / \alpha} L_{1 / n}\right) \\
-\frac{v^{2}}{2 n} h_{1}^{2}\left(n^{1 / \alpha} L_{1 / n}\right)-\frac{w^{2}}{2 n} h_{2}^{2}\left(n^{1 / \alpha} L_{1 / n}\right)-\frac{v w}{n}\left(h_{1} h_{2}\right)\left(n^{1 / \alpha} L_{1 / n}\right)+o(1 / n),
\end{array}
$$


where for any $p \geq 0, o\left(1 / n^{p}\right)$ is a bounded term such that $n^{p} o\left(1 / n^{p}\right) \rightarrow 0$ as $n$ goes to infinity. Consequently we obtain

$$
\begin{array}{r}
\phi_{n}(u, v, w)=\mathbb{E} e^{i u L_{1 / n}}+i \frac{v}{n^{1 / 2}} \mathbb{E} e^{i u L_{1 / n}} h_{1}\left(n^{1 / \alpha} L_{1 / n}\right)+i \frac{w}{n^{1 / 2}} \mathbb{E} e^{i u L_{1 / n}} h_{2}\left(n^{1 / \alpha} L_{1 / n}\right) \\
-\frac{v^{2}}{2 n} \mathbb{E} e^{i u L_{1 / n}} h_{1}^{2}\left(n^{1 / \alpha} L_{1 / n}\right)-\frac{w^{2}}{2 n} \mathbb{E} e^{i u L_{1 / n}} h_{2}^{2}\left(n^{1 / \alpha} L_{1 / n}\right)-\frac{v w}{n} \mathbb{E} e^{i u L_{1 / n}}\left(h_{1} h_{2}\right)\left(n^{1 / \alpha} L_{1 / n}\right)+o(1 / n) .
\end{array}
$$

From this expansion, we have to study the convergence of

1. $\mathbb{E} e^{i u L_{1 / n}}$,

2. $\mathbb{E} e^{i u L_{1 / n}} h\left(n^{1 / \alpha} L_{1 / n}\right)$ for $h$ a bounded function such that $\mathbb{E} h\left(L_{1}^{\alpha}\right)=0$,

3. $\mathbb{E} e^{i u L_{1 / n}} h^{2}\left(n^{1 / \alpha} L_{1 / n}\right)$ for $h$ a bounded function,

4. $\mathbb{E} e^{i u L_{1 / n}}\left(h_{1} h_{2}\right)\left(n^{1 / \alpha} L_{1 / n}\right)$ for $h_{1}, h_{2}$ bounded and such that $\mathbb{E}\left(h_{1} h_{2}\right)\left(L_{1}^{\alpha}\right)=0$

The terms 1., 3., and 4 . are easy to study and only 2 . requires some more work.

Term 1. We have $\mathbb{E} e^{i u L_{1 / n}}=1+\psi(u) / n+o(1 / n)$, where $\psi$ is the Lévy-Khintchine exponent of $L_{1}$.

Term 3. We decuce from Theorem 4.2 that $\mathbb{E} e^{i u L_{1 / n}} h^{2}\left(n^{1 / \alpha} L_{1 / n}\right)=\mathbb{E} e^{i u L_{1}^{\alpha} / n^{1 / \alpha}} h^{2}\left(L_{1}^{\alpha}\right)+o\left(1 / n^{1 / 2}\right)$. Moreover from dominated convergence Theorem, we have

$$
\mathbb{E} e^{i u L_{1}^{\alpha} / n^{1 / \alpha}} h^{2}\left(L_{1}^{\alpha}\right)=\mathbb{E} h^{2}\left(L_{1}^{\alpha}\right)+o(1),
$$

we conclude that

$$
\mathbb{E} e^{i u L_{1 / n}} h^{2}\left(n^{1 / \alpha} L_{1 / n}\right)=\mathbb{E} h^{2}\left(L_{1}^{\alpha}\right)+o(1) .
$$

Term 4. In the same way, we have

$$
\mathbb{E} e^{i u L_{1 / n}\left(h_{1} h_{2}\right)\left(n^{1 / \alpha} L_{1 / n}\right)=o(1) .}
$$

Term 2. Theorem 4.2 yields

$$
\mathbb{E} e^{i u L_{1 / n}} h\left(n^{1 / \alpha} L_{1 / n}\right)=\mathbb{E} e^{i u L_{1}^{\alpha} / n^{1 / \alpha}} h\left(L_{1}^{\alpha}\right)+o\left(1 / n^{1 / 2}\right) .
$$

Now we observe that $\mathbb{E} e^{i u L_{1}^{\alpha} / n^{1 / \alpha}} h\left(L_{1}^{\alpha}\right)=\mathbb{E}\left(e^{i u L_{1}^{\alpha} / n^{1 / \alpha}}-1\right) h\left(L_{1}^{\alpha}\right)$. To control this term, we consider separately the cases $\alpha>1$ and $\alpha \leq 1$.

$\bullet \alpha>1$. Since $\mathbb{E}\left|L_{1}^{\alpha}\right|<\infty$ and $h$ is bounded, we immediately obtain

$$
\left|\mathbb{E}\left(e^{i u L_{1}^{\alpha} / n^{1 / \alpha}}-1\right) h\left(L_{1}^{\alpha}\right)\right| \leq C|u| / n^{1 / \alpha}=o\left(1 / n^{1 / 2}\right) .
$$

$\bullet \alpha \leq 1$. From the Lévy-Itô representation, we have

$$
L_{1}^{\alpha}=L_{1}^{\alpha, 1}+L_{1}^{\alpha, 2},
$$

where

$$
L_{1}^{\alpha, 1}=\int_{0}^{1} \int_{\{0<|z| \leq 1\}} z \tilde{\mu}^{\alpha}(d t, d z), \quad L_{1}^{\alpha, 2}=\int_{0}^{1} \int_{\{|z|>1\}} z \mu^{\alpha}(d t, d z),
$$

$\mu^{\alpha}$ is a Poisson random measure with compensator $d t \frac{1}{|z|^{\alpha+1}} d z$ and $\tilde{\mu}^{\alpha}$ is the compensated measure. With these notations, we set

$$
A_{n}=\left\{\mu^{\alpha}\left([0,1] \times\left\{|z|>n^{1 / \alpha}\right\}\right)=0\right\} .
$$

Since $\mu^{\alpha}\left([0,1] \times\left\{|z|>n^{1 / \alpha}\right\}\right)$ has a Poisson distribution with parameter bounded by $C / n$, we deduce that

$$
\mathbb{P}\left(A_{n}^{c}\right) \leq C / n .
$$

Using the truncation $1_{A_{n}}$, this permits to get the bound

$$
\left|\mathbb{E}\left(e^{i u L_{1}^{\alpha} / n^{1 / \alpha}}-1\right) h\left(L_{1}^{\alpha}\right)\right| \leq C \mathbb{E}\left|e^{i u L_{1}^{\alpha} / n^{1 / \alpha}}-1\right| 1_{A_{n}}+C / n \leq C \mathbb{E}\left(\left|L_{1}^{\alpha}\right| 1_{A_{n}}\right) / n^{1 / \alpha}+C / n .
$$


Obviously we have $\mathbb{E}\left|L_{1}^{\alpha, 1}\right| \leq C$ and for the big jumps component we get

$$
\mathbb{E}\left|L_{1}^{\alpha, 2}\right| 1_{A_{n}} \leq \int_{\left\{1<|z| \leq n^{1 / \alpha}\right\}} \frac{|z|}{|z|^{\alpha+1}} d z \leq\left\{\begin{array}{l}
C \log n, \text { if } \alpha=1 \\
C n^{1 / \alpha} / n \text { if } \alpha<1 .
\end{array}\right.
$$

This leads to

$$
\left|\mathbb{E}\left(e^{i u L_{1}^{\alpha} / n^{1 / \alpha}}-1\right) h\left(L_{1}^{\alpha}\right)\right| \leq\left\{\begin{array}{l}
C \log n / n, \text { if } \alpha=1 \\
C / n \text { if } \alpha<1
\end{array}\right.
$$

In both cases, we conclude for the term 2. that

$$
\left|\mathbb{E} e^{i u L_{1 / n}} h\left(n^{1 / \alpha} L_{1 / n}\right)\right| \leq o\left(1 / n^{1 / 2}\right) .
$$

Putting all these results together, we finally obtain the convergence

$$
\log \Phi_{n}(u, v, w)=n \log \phi_{n}(u, v, w) \rightarrow \psi(u)-\frac{v^{2}}{2} \mathbb{E} h_{1}^{2}\left(L_{1}^{\alpha}\right)-\frac{w^{2}}{2} \mathbb{E} h_{2}^{2}\left(L_{1}^{\alpha}\right)
$$

and we get the convergence in law of the vector $\left(\bar{L}_{1}^{n}, \Gamma_{1}^{\prime n}\right)$ to $\left(L_{1}, \gamma^{\prime}\right)$ where $\gamma^{\prime}$ is a Gaussian variable independent of $L_{1}$ with variance $\Sigma^{\prime}$ defined by (5.12).

This achieves the proof of Theorem 3.2 .

\section{Proof of Corollary 3.1.}

From Theorem 3.2, it is sufficient to prove that for $f: \mathbb{R} \mapsto \mathbb{R}$ a continuous function and $h: \mathbb{R} \mapsto \mathbb{R}$ a bounded function with bounded derivative

$$
\frac{1}{n^{1 / 2}} \sum_{i=1}^{n} f\left(X_{\frac{i-1}{n}}, \theta_{0}, \sigma_{0}\right)\left(h\left(z_{n}\left(X_{\frac{i-1}{n}}, X_{\frac{i}{n}}, \theta_{0}, \sigma_{0}\right)\right)-h\left(n^{1 / \alpha} \Delta L_{i}\right)\right) \rightarrow 0, \quad \text { in probability. }
$$

For this we check the conditions (5.1) and (5.2) with

$$
\zeta_{i}^{n}=\frac{1}{n^{1 / 2}} f\left(X_{\frac{i-1}{n}}, \theta_{0}, \sigma_{0}\right)\left(h\left(z_{n}\left(X_{\frac{i-1}{n}}, X_{\frac{i}{n}}, \theta_{0}, \sigma_{0}\right)\right)-h\left(n^{1 / \alpha} \Delta L_{i}\right)\right)
$$

From Theorem 4.1

$$
\left|\mathbb{E}_{\mid \mathcal{F}_{\frac{i-1}{n}}} h\left(z_{n}\left(X_{\frac{i-1}{n}}, X_{\frac{i}{n}}, \theta_{0}, \sigma_{0}\right)\right)-\mathbb{E}_{\mid \mathcal{F}_{\frac{i-1}{n}}} h\left(n^{1 / \alpha} \Delta L_{i}\right)\right| \leq C\left(1+\left|X_{\frac{i-1}{n}}\right|\right) \varepsilon_{n}\|h\|_{\infty},
$$

where $n^{1 / 2} \varepsilon_{n} \rightarrow 0$ and (5.1) is immediate. Turning to (5.2) and using that $h$ and $h^{\prime}$ are bounded, we have for all $\varepsilon>0$

$$
\mathbb{E}_{\mid \mathcal{F}_{\frac{i-1}{n}}}\left|h\left(z_{n}\left(X_{\frac{i-1}{n}}, X_{\frac{i}{n}}, \theta_{0}, \sigma_{0}\right)\right)-h\left(n^{1 / \alpha} \Delta L_{i}\right)\right|^{2} \leq C \varepsilon^{2}+C \mathbb{E}_{\mid \mathcal{F}_{\frac{i-1}{n}}} 1_{\left.\left\{\mid z_{n}\left(X_{\frac{i-1}{n}}, X_{\frac{i}{n}}, \theta_{0}, \sigma_{0}\right)\right)-n^{1 / \alpha} \Delta L_{i} \mid>\varepsilon\right\}}
$$

From Lemma 4.2, $\forall \varepsilon>0, \mathbb{E}_{\mid \mathcal{F}_{\frac{i-1}{n}}} 1_{\left.\left\{\mid z_{n}\left(X_{\frac{i-1}{n}}, X_{\frac{i}{n}}, \theta_{0}, \sigma_{0}\right)\right)-n^{1 / \alpha} \Delta L_{i} \mid>\varepsilon\right\}} \leq C(\varepsilon)\left(1+\left|X_{\frac{i-1}{n}}\right|^{p}\right) \frac{1}{n^{q}}$ for $p, q>0$ and we deduce

$$
\limsup _{n} \frac{1}{n} \sum_{i=1}^{n} f^{2}\left(X_{\frac{i-1}{n}}, \theta_{0}, \sigma_{0}\right) \mathbb{E}_{\mid \mathcal{F}_{\frac{i-1}{n}}} 1_{\left.\left\{\mid z_{n}\left(X_{\frac{i-1}{n}}, X_{\frac{i}{n}}, \theta_{0}, \sigma_{0}\right)\right)-n^{1 / \alpha} \Delta L_{i} \mid>\varepsilon\right\}}=0 \quad \text { a.s. }
$$

This yields $\lim \sup _{n} \sum_{i=1}^{n} \mathbb{E}_{\mid \mathcal{F}_{\frac{i-1}{n}}}\left|\zeta_{i}^{n}\right|^{2} \leq C \varepsilon^{2}$, a.s. and we get (5.2) letting $\varepsilon$ go to zero. 


\section{References}

[1] Aït-Sahalia, Y. and Jacod, J. (2007). Volatility estimators for discretely sampled Lévy processes. Ann. Statist. 35 355-392. MR2332279

[2] Aït-Sahalia, Y. and JACOD, J. (2008). Fisher's information for discretely sampled Lévy processes. Econometrica $\mathbf{7 6}$ 727-761. MR2433480 (2010g:60088)

[3] Aїт-Sahalia, Y. and Jacod, J. (2009). Estimating the degree of activity of jumps in high frequency data. Ann. Statist. 37 2202-2244. MR2543690

[4] Barndorff-Nielsen, O. and Sørensen, M. (1994). A review of some aspects of asymptotic likelihood theory for stochastic processes. International Statistical Review 62 133-165.

[5] Bibby, B. M. and Sørensen, M. (1995). Martingale estimation functions for discretely observed diffusion processes. Bernoulli 1 17-39. MR1354454

[6] Bichteler, K., Gravereaux, J.-B. and Jacod, J. (1987). Malliavin calculus for processes with jumps. Stochastics Monographs 2. Gordon and Breach Science Publishers, New York. MR1008471 (90h:60056)

[7] Clément, E. and Gloter, A. (2015). Local asymptotic mixed normality property for discretely observed stochastic differential equations driven by stable Lévy processes. Stochastic Process. Appl. 125 2316-2352. MR3322866

[8] Clément, E., Gloter, A. and Nguyen, H. (2018a). LAMN property for the drift and volatility parameters of a SDE driven driven by a stable Lévy process. ESAIM Probab. Stat.

[9] Clément, E., Gloter, A. and Nguyen, H. (2018b). Asymptotics in small time for the density of a stochastic differential equation driven driven by a stable Lévy process. ESAIM Probab. Stat.

[10] Ibragimov, I. A. and Has'minskiĬ, R. (1981). Statistical estimation. Applications of Mathematics 16. Springer-Verlag, New York-Berlin Asymptotic theory, Translated from the Russian by Samuel Kotz. MR620321

[11] Ivanenko, D., Kulik, A. M. and Masuda, H. (2015). Uniform LAN property of locally stable Lévy process observed at high frequency. ALEA Lat. Am. J. Probab. Math. Stat. 12 835-862. MR3453298

[12] JACOD, J. (2004). The Euler scheme for Lévy driven stochastic differential equations: limit theorems. Ann. Probab. 32 1830-1872. MR2073179 (2005f:65013)

[13] Jacod, J. and Protter, P. (2012). Discretization of processes. Stochastic Modelling and Applied Probability 67. Springer, Heidelberg. MR2859096

[14] Jacod, J. and Sørensen, M. (2018). A review of asymptotic theory of estimating functions. Stat. Inference Stoch. Process.

[15] Kawai, R. and Masuda, H. (2011). On the local asymptotic behavior of the likelihood function for Meixner Lévy processes under high-frequency sampling. Statist. Probab. Lett. 81 460-469. MR2765166

[16] Kawai, R. and Masuda, H. (2013). Local asymptotic normality for normal inverse Gaussian Lévy processes with highfrequency sampling. ESAIM Probab. Stat. 17 13-32. MR3002994

[17] Kessler, M. and Sørensen, M. (1999). Estimating equations based on eigenfunctions for a discretely observed diffusion process. Bernoulli 5 299-314. MR1681700

[18] KuLIK, A. (2015). On weak uniqueness and distributional properties of a solution to an SDE with $\alpha$-stable noise. arXiv: $1511.00106 . v 1$.

[19] Luschgy, H. and Pagès, G. (2008). Moment estimates for Lévy processes. Electron. Commun. Probab. $13422-434$. MR2430710

[20] Masuda, H. (2009). Joint estimation of discretely observed stable Lévy processes with symmetric Lévy density. J. Japan Statist. Soc. 39 49-75. MR2571802

[21] Masuda, H. (2018). Non-gaussian quasi-likelihood estimation of SDE driven by locally stable Lévy process. to appear in Stochastic Process. Appl.

[22] Meyer, P. A. (1972). Les résultats récents de Burkholder, Davis et Gundy. 151-158. Lecture Notes in Math., Vol. 258. MR0383520

[23] Sørensen, M. (1999). On asymptotics of estimating functions. Braz. J. Probab. Stat. 13 111-136. MR1803041

[24] Todorov, V. (2015). Jump activity estimation for pure-jump semimartingales via self-normalized statistics. Ann. Statist. 43 1831-1864. MR3357880

[25] Todorov, V. and Tauchen, G. (2011). Limit theorems for power variations of pure-jump processes with application to activity estimation. Ann. Appl. Probab. 21 546-588. MR2807966 